\title{
POLYHARMONIC FUNCTIONS ON TREES
}

\author{
By Joel M. Cohen, Flavia Colonna, Kohur Gowrisankaran, \\ and DAVID Singman
}

\begin{abstract}
In this paper, we introduce and study polyharmonic functions on trees. We prove that the discrete version of the classical Riquier problem can be solved on trees. Next, we show that the discrete version of a characterization of harmonic functions due to Globevnik and Rudin holds for biharmonic functions on trees. Furthermore, on a homogeneous tree we characterize the polyharmonic functions in terms of integrals with respect to finitely-additive measures (distributions) of certain functions depending on the Poisson kernel. We define polymartingales on a homogeneous tree and show that the discrete version of a characterization of polyharmonic functions due to Almansi holds for polymartingales. We then show that on homogeneous trees there are 1-1 correspondences among the space of $n$ th-order polyharmonic functions, the space of $n$ th-order polymartingales, and the space of $n$-tuples of distributions. Finally, we study the boundary behavior of polyharmonic functions on homogeneous trees whose associated distributions satisfy various growth conditions.
\end{abstract}

1. Introduction. Polyharmonic functions on a domain in $\mathbb{R}^{N}$ are solutions to the differential equation $\Delta^{n}=0$, for some $n \in \mathbb{N}$, where $\Delta$ is the Laplace operator. These functions have been studied extensively by Almansi in [1] and [2]. The biharmonic case $-n=2$-arises naturally in connection with problems in the theory of elasticity (cf. [25], [22]) and in radar imaging (cf. [3]). There is a vast literature on polyharmonic functions. For basic references, see [25] and [4].

In recent years there has been considerable attention given to discretizations of many classical problems in harmonic analysis and geometry. The groundwork for the study of functions on trees was laid out by Cartier in [7]. Recent developments of the study of harmonic analysis, functional analysis and potential theory on trees include, among many other works, [17], [21], [27], [14], [5], [11], [10], [12], [13], [9], [15], [19]. Discretizations of harmonic and biharmonic functions on certain graphs with the purpose of solving differential equations on fractals such as the Sierpinski gasket are beginning to appear in the literature (cf. for example, [20], [16]).

In this article, we define and study polyharmonic functions on trees. We use [7] as a general reference on trees.

A tree is a locally finite connected graph with no loops, which, as a set, is identified with the collection of its vertices. A graph contained in a tree is called a subtree. Two vertices $v$ and $w$ of a tree are called neighbors if there is an edge connecting them, in which case we use the notation $v \sim w$. A path is a finite

Manuscript received February 15, 2001.

American Journal of Mathematics 124 (2002), 999-1043. 
or infinite sequence of vertices $\left[v_{0}, v_{1}, \ldots\right]$ such that $v_{k} \sim v_{k+1}$ and $v_{k-1} \neq v_{k+1}$ for all $k$. If $u$ and $v$ are any vertices, we denote by $[u, v]$ the unique path joining them.

Fixing $e$ as a root of the tree, the predecessor $u^{-}$of a vertex $u$, with $u \neq e$, is the next to the last vertex of the path from $e$ to $u$. An ancestor of $u$ is any vertex in the path from $e$ to $u^{-}$. By convention, we set $e^{-}=e$. We call children of a vertex $v$ the vertices $u$ such that $u^{-}=v$.

A tree $T$ may be endowed with a metric $d$ as follows. If $u, v$ are vertices, $d(u, v)$ is the number of edges in the unique path from $u$ to $v$. Given a root $e$, the length of a vertex $v$ is defined as $|v|=d(e, v)$.

Given a tree $T$, let $p$ be a nearest-neighbor transition probability on the vertices of $T$, that is, $p(v, u)>0$, if $v$ and $u$ are neighbors, $p(v, u)=0$, if $v$ and $u$ are not neighbors. It is convenient to set $p(v, v)=-1$, so that for each vertex $v$, we have $\sum_{u} p(v, u)=0$.

As is customary, a function on a tree $T$ will mean a function on its set of vertices. The Laplacian of a function $f: T \rightarrow \mathbb{C}$ is defined as

$$
\Delta f(v)=\sum_{u \in T} p(v, u) f(u) \text { for all nonterminal vertices } v \in T,
$$

where by terminal we mean a vertex which has only one neighbor. A function $f$ on $T$ is said to be harmonic if its Laplacian is identically zero.

The boundary $\Omega$ of $T$ is the set of equivalence classes of infinite paths under the relation $\simeq$ defined by the shift, $\left[v_{0}, v_{1}, \ldots\right] \simeq\left[v_{1}, v_{2}, \ldots\right]$, together with the set of terminal vertices. For any vertex $u$, we denote by $[u, \omega)$ the (unique) path starting at $u$ in the class $\omega$. Then $\Omega$ can be identified with the set of paths starting at $u$. Furthermore, $\Omega$ is a compact space under the topology generated by the sets

$$
I_{v}^{u}=\{\omega \in \Omega: v \in[u, \omega)\}
$$

A distribution is a finitely additive complex measure on finite unions of the sets $I_{v}^{u}$.

Each $\omega \in \Omega$ induces an orientation on the edges of $T:[u, v]$ is positively oriented if $v \in[u, \omega)$. For $\omega \in \Omega$, and $u, v \in T$, define the horocycle index $k_{\omega}(u, v)$ as the number of positively oriented edges minus the number of negatively oriented edges in the path from $u$ to $v$.

By a homogeneous tree of degree $q+1$ (with $q \geq 2$ ) we mean a tree $T$ whose vertices have $q+1$ neighbors and whose associated nearest-neighbor transition probability is $p(v, u)=\frac{1}{q+1}$ if $v$ and $u$ are neighbors.

For a homogeneous tree of degree $q+1$, the Poisson kernel (with respect to the vertex $u$ ) is then given by

$$
P_{\omega}(u, v)=q^{k_{\omega}(u, v)}
$$


since it satisfies the following properties analogous to those that hold in the classical case [27]:

(i) For fixed $u \in T$ and $\omega \in \Omega, v \mapsto P_{\omega}(u, v)$ is a harmonic function on $T$ (the value at any vertex is the average of the values at its neighbors).

(ii) If $\mu$ is a distribution, then the function defined by the Poisson integral with respect to $u$

$$
f(v)=\int_{\Omega} P_{\omega}(u, v) d \mu(\omega)
$$

is well defined and harmonic on $T$. Conversely, fixing $u \in T$, every harmonic function $f$ on $T$ has such an integral representation for some unique distribution $\mu$.

A Poisson kernel can be defined also for nonhomogeneous trees, and (i) and (ii) hold in general. Some of the results of this paper hold only for homogeneous trees. Whenever we use the terminology "general tree" in a definition or result, we emphasize that homogeneity is not required.

Definition 1.1. Let $T$ be a general tree. For $n \in \mathbb{N}$, a function $f: T \rightarrow \mathbb{C}$ is polyharmonic of order $n$ at $v \in T$ if $\Delta^{n} f(v)=0$, where $\Delta^{n}$ is the $n$-fold composition of $\Delta$. A polyharmonic function of order $n$ on $T$ is a function which is polyharmonic of order $n$ at every vertex of $T$. Thus, a polyharmonic function of order one is harmonic. A polyharmonic function of order 2 is called biharmonic. We shall also call a polyharmonic function of order $n, n$-polyharmonic.

We now outline the main results of the paper. In Section 2, we consider the discrete Riquier problem of determining a polyharmonic function $f$ of order $n$ on a finite complete connected subtree of a general tree (see Definition 2.1) with prescribed values $f, \Delta f, \Delta^{2} f, \ldots, \Delta^{n-1} f$ on the boundary of the subtree. In Theorem 2.1 we give the formula for the solution in the biharmonic case in terms of the transition probabilities. We then provide explicit formulas for the case when the subtree is a disk or a tube.

In Section 3 (Theorem 3.1), we prove the following result which was inspired by a classical theorem for harmonic functions due to Globevnik and Rudin (cf. [18]): Given a function $f$ defined on a general tree $T$ and a fixed vertex $e, f$ is biharmonic on $T$ if it has the property that for every tube (see Definition 2.2) whose interior contains $e$, the solution to the Riquier problem with boundary values $f$ and $\Delta f$ agrees with $f$ at $e$. The methods used in proving this theorem yield a very simple proof, which we include, of the analogous characterization of harmonic functions on trees proved originally in [5].

In Sections 4, 5 and 6, we restrict our attention to homogeneous trees with a fixed root $e$.

In Section 4 (Theorem 4.1), we give an integral representation formula in the spirit of a formula due to Almansi in the classical case, for all polyharmonic functions. Specifically, to every $n$-polyharmonic function $f$ there correspond unique 
distributions $\nu_{0}, \ldots, \nu_{n-1}$ such that $f=\sum_{j=0}^{n-1} k^{j} P \nu_{j}$, where

$$
k^{j} P \nu_{j}(v)=\int_{\Omega} k_{\omega}(e, v)^{j} P_{\omega}(e, v) d \nu_{j}(\omega)
$$

In Section 5, we define polymartingales on a homogeneous tree and in Theorem 5.1 we prove the discrete version of Almansi's Theorem for polymartingales, which is the precise counterpart of the classical Almansi formula for polyharmonic functions. This suggests that in many cases it is more natural to study polymartingales than polyharmonic functions on homogeneous trees. We then show that there are one-to-one correspondences among the space of $n$-polyharmonic functions, the space of $n$-polymartingales, and the space of $n$-tuples of distributions, thus extending similar correspondences among the spaces of harmonic functions, martingales, and distributions given in [27].

In Section 6, we study the boundary behavior of polyharmonic functions. Specifically, in Theorem 6.1 we show that given a positive measure $\nu$ on the Borel sets of $\Omega$, the function $v \mapsto k^{n} P \nu(v) /|v|^{n}$ has a nontangential limit at $\mu^{e}$ a.e. boundary point of $\Omega$, where $\mu^{e}$ denotes the natural probability measure on $\Omega$ with respect to $e$. We also show that in the special case where $\nu$ is absolutely continuous with respect to $\mu^{e}$ and the associated density function is continuous, the above limit is unrestricted.

Given $0<\beta \leq 1$ and $\tau \geq 1$, we recall the definitions of $\beta$-dimensional Hausdorff measure on $\Omega$ and of $\tau$-tangential approach regions introduced in [19]. We then consider $n$-polyharmonic functions whose representing distributions satisfy certain growth conditions depending on $\beta$ and $\tau$. In Theorem 6.2, we show that for appropriate values of $\beta$ and $\tau$, the function $v \mapsto f(v) /|v|^{n-1}$ has a limit at every boundary point with the exception of a subset of $\beta$-dimensional Hausdorff measure zero, if approach is restricted to the $\tau$-tangential region. In Theorem 6.3, we show that the exceptional sets and approach regions in Theorem 6.2 are the best possible.

Sections 4, 5, and 6 can be read independently of the other sections.

Acknowledgment. The authors wish to thank Ibtesam Bajunaid for bringing the topic of biharmonic functions to their attention.

2. The Riquier problem on trees. The classical Riquier problem consists of determining a polyharmonic function $f$ of order $n$ on a domain $D \subset \mathbb{R}^{d}$, with prescribed values on $\partial D$ of the functions $f, \Delta f, \Delta^{2} f, \ldots, \Delta^{n-1} f$. For the case $n=2$ the problem was considered by Mathieu [23], and in general by Riquier [26].

In this section we solve the discrete counterpart of the classical Riquier problem for biharmonic functions on general trees. It is straightforward to generalize statements and results to polyharmonic functions of higher order. 
Definition 2.1. Given a finite subtree $S$ of $T$, we define the interior of $S$ as the set $\stackrel{\circ}{S}$ consisting of all vertices $v \in S$ such that every vertex of $T$ which is a neighbor of $v$ belongs to $S$. The boundary of $S$ in $T$ is defined as the set $\partial S$ of all vertices $v \in S$ such that exactly one neighbor of $v$ is in $S$. We say that $S$ is a complete subtree of $T$ if $S=\stackrel{\circ}{S} \cup \partial S$.

Given $S$ a complete subtree of $T$, if $f$ is a function defined on $\stackrel{\circ}{S}$ and $g$ is a function defined on $\partial S$, then $\Delta(f \cup g)$ is well defined on $\stackrel{\circ}{S}$, where

$$
(f \cup g)(v)= \begin{cases}f(v) & \text { if } v \in \stackrel{\circ}{S} \\ g(v) & \text { if } v \in \partial S\end{cases}
$$

We say that $f$ is harmonic on $S$ with boundary values $g$, and use the notation $f \mid \partial S=g$, if $\Delta(f \cup g)=0$ on $\stackrel{\circ}{S}$.

Similarly, if $f$ is defined on $\stackrel{\circ}{S}$ and $g_{1}$ and $g_{2}$ are functions defined on $\partial S$, then $\Delta\left[\Delta\left(f \cup g_{1}\right) \cup g_{2}\right]$ is well defined on $\stackrel{\circ}{S}$. If this is identically zero on $\stackrel{\circ}{S}$, we say that $f$ is biharmonic on $S$ with boundary values $g_{1}$ and $g_{2}$ and we use the notations $f \mid \partial S=g_{1}$ and $\Delta f \mid \partial S=g_{2}$.

Discrete Riquier Problem. Let $T$ be a tree, and let $S$ be a finite complete connected subtree of $T$. Given two functions $g_{1}$ and $g_{2}$ on $\partial S$, find a function $f$ on $S$ biharmonic on $\stackrel{\circ}{S}$ such that $f \mid \partial S=g_{1}$ and $\Delta f \mid \partial S=g_{2}$.

For $S$ a finite complete connected subtree of $T$, it is possible to solve the discrete Dirichlet problem (cf. [5]): given a function $g$ on $\partial S$, there exists a function $f$ harmonic on $S$ such that $f \mid \partial S=g$. Although we will not use it directly, it is worth mentioning how the construction works: for $v \in \stackrel{\circ}{S}$ and $u \in \partial S$, let $P_{S}(v, u)$ be the probability that a path starting at $v$ reaches $u$ before reaching any other point of $\partial S$. The function $P_{S}$ is called the Poisson kernel with respect to $S$. Then the solution to the Dirichlet problem is given by

$$
f(v)=\sum_{u \in \partial S} P_{S}(v, u) g(u)
$$

The solution is necessarily unique. Indeed, since the value at each point is the average of the values at its neighbors, the maximum and minimum cannot be reached at an interior point unless the function is constant. Thus the only solution to the Dirichlet problem with boundary values zero is necessarily the trivial function.

The uniqueness of the solution to the Dirichlet problem easily implies the uniqueness of the solution to the Riquier problem. 
Let $S$ be a finite complete connected subtree of a tree $T$ not necessarily homogeneous. Let $m$ be the cardinality of $S$ and let $s$ be the number of its interior vertices. Label the interior vertices of $S v_{j}, 1 \leq j \leq s$, and the vertices of $\partial S v_{k}$, $s+1 \leq k \leq m$. Let $P$ be the $s \times s$ matrix with entries $p\left(v_{i}, v_{j}\right)$ for $1 \leq i, j \leq s$, and let $Q$ be the $s \times(m-s)$ matrix with entries $p\left(v_{i}, v_{k}\right)$ for $1 \leq i \leq s$ and $s+1 \leq j \leq m$. Given a function $f$ on the interior of $S$ and a function $g$ on the boundary of $S$, let $\mathbf{f}$ and $\mathbf{g}$ be the column vectors with entries $f\left(v_{j}\right), 1 \leq j \leq m$, and $g\left(v_{k}\right), s+1 \leq k \leq m$, respectively. Then $h=f \cup g$ is defined on $S$, and its Laplacian is defined on the interior of $\mathrm{S}$ and is given by

$$
\Delta h=P \mathbf{f}+Q \mathbf{g} .
$$

In particular, we see that the solution to the Dirichlet problem for $g$ is given by a function $f$ such that $0=P \mathbf{f}+Q \mathbf{g}$. By the uniqueness of the solution, $P$ must be invertible, and so the solution to the Dirichlet problem is given by

$$
\mathbf{f}=-P^{-1} Q \mathbf{g}
$$

THEOREM 2.1. The solution to the Riquier problem with boundary values $g_{1}$ and $g_{2}$ exists and is given by

$$
\mathbf{f}=-P^{-1} Q \mathbf{g}_{1}-\left(P^{-1}\right)^{2} Q \mathbf{g}_{2} .
$$

Proof. Let $h$ be the solution to the Dirichlet problem with boundary values $g_{2}$, that is, $\mathbf{h}=-P^{-1} Q \mathbf{g}_{2}$, and let

$$
\mathbf{f}=-P^{-2} Q \mathbf{g}_{2}-P^{-1} Q \mathbf{g}_{1}
$$

Then

$$
\Delta\left(f \cup g_{1}\right)=P \mathbf{f}+Q \mathbf{g}_{1}=-P^{-1} Q \mathbf{g}_{2}-Q \mathbf{g}_{1}+Q \mathbf{g}_{1}=\mathbf{h}
$$

and so $\Delta f$ is the required harmonic function with boundary values $g_{2}$.

We next derive the explicit formulas for the matrices $P$ and $Q$ in the homogeneous case when $S$ is either a disk or a tube.

Example 2.1. Let $T$ be a homogeneous tree of degree $q+1$. For $n \in \mathbb{N}$ and $v \in T$, let $B_{n}(v)=\{u \in T: d(u, v) \leq n\}$, the disk centered at $v$ of radius $n$. Let $t$ and $s$ be the number of boundary vertices and the number of interior vertices in $B_{n}(v)$, respectively. Thus

$$
t=t_{n}=(q+1) q^{n-1}, s=s_{n}=\frac{q^{n}+q^{n-1}-2}{q-1} .
$$


Order the vertices in $B_{n}(v)$ according to increasing length and by labeling the descendants of each vertex sequentially and consistently throughout the process. Then the $s \times s$ matrix $P$ is symmetric, all the diagonal entries are -1 , the top $s_{n-1}$ rows contain $q+1$ terms $\frac{1}{q+1}$, while the remaining rows contain a single entry $\frac{1}{q+1}$ and all other entries (except for the diagonal entry) are zero. The $s \times t$ matrix $Q$ consists of $s_{n-1}$ rows of zeros followed by $t_{n-1}$ rows in echelon form each of which consists of a block of $q$ entries $\frac{1}{q+1}$ and all other entries zero.

Specifically, for the case $q=2$ and $n=2$ the matrices $P$ and $Q$ are given by

$$
P=\left[\begin{array}{cccc}
-1 & \frac{1}{3} & \frac{1}{3} & \frac{1}{3} \\
\frac{1}{3} & -1 & 0 & 0 \\
\frac{1}{3} & 0 & -1 & 0 \\
\frac{1}{3} & 0 & 0 & -1
\end{array}\right]
$$

and

$$
Q=\left[\begin{array}{cccccc}
0 & 0 & 0 & 0 & 0 & 0 \\
\frac{1}{3} & \frac{1}{3} & 0 & 0 & 0 & 0 \\
0 & 0 & \frac{1}{3} & \frac{1}{3} & 0 & 0 \\
0 & 0 & 0 & 0 & \frac{1}{3} & \frac{1}{3}
\end{array}\right]
$$

Hence, the matrices that yield the explicit solution of the Riquier problem are

$$
-P^{-1} Q=\frac{1}{18}\left[\begin{array}{llllll}
3 & 3 & 3 & 3 & 3 & 3 \\
7 & 7 & 1 & 1 & 1 & 1 \\
1 & 1 & 7 & 7 & 1 & 1 \\
1 & 1 & 1 & 1 & 7 & 7
\end{array}\right]
$$

and

$$
\left(P^{-1}\right)^{2} Q=\frac{1}{18}\left[\begin{array}{cccccc}
9 & 9 & 9 & 9 & 9 & 9 \\
10 & 10 & 4 & 4 & 4 & 4 \\
4 & 4 & 10 & 10 & 4 & 4 \\
4 & 4 & 4 & 4 & 10 & 10
\end{array}\right]
$$

Definition 2.2. A tube is a complete finite subtree whose interior is a path. 
Example 2.2. Let $T$ be a homogeneous tree of degree $q+1$, and let $S$ be a tube in $T$ whose interior is the path $\left[v_{1}, \ldots, v_{k}\right]$. Then $P$ is the symmetric $k \times k$ matrix

$$
P=\left[\begin{array}{cccccc}
-1 & \frac{1}{q+1} & 0 & 0 & \cdots & 0 \\
\frac{1}{q+1} & -1 & \frac{1}{q+1} & 0 & \cdots & 0 \\
0 & \frac{1}{q+1} & -1 & \frac{1}{q+1} & \cdots & 0 \\
\vdots & \vdots & \vdots & \ddots & \vdots & \vdots \\
0 & \cdots & 0 & \frac{1}{q+1} & -1 & \frac{1}{q+1} \\
0 & \cdots & 0 & 0 & \frac{1}{q+1} & -1
\end{array}\right]
$$

while $Q$ is the $k \times(k q-k+2)$ matrix in echelon form whose first and last row consist of a block of $q$ entries $\frac{1}{q+1}$ and all other entries zero and the intermediate rows consist of a block of $q-1$ entries $\frac{1}{q+1}$ and all other entries zero.

This matrix $P$ is a type of symmetric matrix that arises in the context of solving a second order difference equation with a given boundary condition. The inverse of $P$ can be found by means of the discrete sine transform (cf. [6], Section 7.1): $P^{-1}=\left[u_{n, m}\right]$, where

$$
u_{n, m}=\frac{2}{k+1} \sum_{j=1}^{k} \frac{\sin \left(\frac{\pi n j}{k+1}\right) \sin \left(\frac{\pi m j}{k+1}\right)}{\frac{2}{q+1} \cos \left(\frac{\pi j}{k+1}\right)-1} .
$$

3. Globevnik-Rudin characterization of biharmonic functions. Let $T$ be a general tree, and let $S=\mathcal{T}\left[v_{1}, \ldots, v_{k}\right]$ be a tube in $T$ (see Definition 2.2) whose interior is the path $\left[v_{1}, \ldots, v_{k}\right]$. Let $f$ be a function defined on $T$, and let $f_{S}$ be the solution to the Dirichlet problem on $S$ with boundary values $f$ on $\partial S$, that is, $\Delta f_{S}=0$ in $\stackrel{\circ}{S}$ and $f_{S}|\partial S=f| \partial S$. Similarly, let us define $f^{S}$ as the solution to the Riquier problem on $S$ with boundary functions $f$ and $\Delta f$ on $\partial S$.

In [5], the authors prove the following discrete version of Globevnik-Rudin characterization of harmonic functions (Theorem 2 of [18]).

THEOREM 4.6 (of [5]). Fix a vertex $e \in T$ and let $f$ be a function on $T$. If for all tubes $S$ whose interior contains e we have $f_{S}(e)=f(e)$, then $f$ is harmonic on $T$.

We shall obtain a similar characterization of biharmonic functions. We shall also present a much simpler proof of Theorem 4.6 of [5] which was derived in our search for a proof of our theorem for the biharmonic case.

THEOREM 3.1. Let e be a fixed vertex of a general tree $T$ and let $f$ be a function on $T$. If for all tubes $S$ whose interior contains e we have $f^{S}(e)=f(e)$, then $f$ is biharmonic on $T$. 
First we describe the method and the notation that we shall use to prove our result.

Let $T$ be a tree and let $e$ be a fixed vertex of $T$. Let $\left[e_{1}, e_{2}, \ldots\right)$ be an infinite path with $e_{1}=e$. For $k$ a positive integer, let $T_{k}$ be the tube $\mathcal{T}\left[e_{1}, \ldots, e_{k}\right]$ and let $\left\{u_{i}\right\}$ be the collection of all neighbors of the vertices $e_{j}$ other than the $e_{j}$ themselves, so that $\partial T_{k}$ is the union of a subset of $\left\{u_{i}\right\}$ with $\left\{e_{k+1}\right\}$. Let $p$ be a nearest-neighbor transition probability on $T$ and set $p(j, l)=p\left(e_{j}, u_{l}\right)$, $p_{j}=p\left(e_{j}, e_{j-1}\right)$, and $q_{j}=p\left(e_{j-1}, e_{j}\right)$, and $p_{1}=q_{1}=0$.

Let $f$ be a function on $T$. Then

$$
\Delta f\left(e_{j}\right)=\sum_{l} p(j, l) f\left(u_{l}\right)+p_{j} f\left(e_{j-1}\right)+q_{j+1} f\left(e_{j+1}\right)-f\left(e_{j}\right)
$$

Let $f_{k}=f_{T_{k}}$, the solution to the Dirichlet problem on $T_{k}$ with boundary values $f \mid \partial T_{k}$. Denote by $D_{j}^{k} f$ the difference $f_{k}\left(e_{j}\right)-f\left(e_{j}\right)$ and denote by $D_{j}^{k} \Delta f$ the difference $\Delta f_{k}\left(e_{j}\right)-\Delta f\left(e_{j}\right)$.

Using (1) and the fact that $f$ and $f_{k}$ agree on $\left\{u_{j}\right\} \cup\left\{e_{k+1}\right\}$, we obtain

$$
D_{j}^{k} \Delta f=p_{j} D_{j-1}^{k} f+q_{j+1} D_{j+1}^{k} f-D_{j}^{k} f, \quad j=1, \ldots, k,
$$

and $D_{0}^{k} f=D_{k+1}^{k} f=0$ (where we regard $e_{0}$ to be one of the vertices $u_{j}$ ). In addition, we define $D_{-1}^{k} f=D_{k+2}^{k} f=0$.

We now give a new proof of the discrete Globevnik-Rudin characterization of harmonic functions.

Proof of Theorem 4.6 of [5]. Using the above notation, let $T_{k}$ be any tube starting at $e=e_{1}$. The hypothesis $f_{S}(e)=f(e)$ means that $D_{1}^{k} f=0$ for all $k \in \mathbb{N}$, where $D_{j}^{k} f=f_{k}\left(e_{j}\right)-f\left(e_{j}\right)$, and $D_{j}^{k} \Delta f=\Delta f_{k}\left(e_{j}\right)-\Delta f\left(e_{j}\right)$. Since $f_{k}$ is harmonic, for all $j \leq k, D_{j}^{k} \Delta f=-\Delta f\left(e_{j}\right)$. We shall prove that $\Delta f\left(e_{j}\right)=0$ for all $j \in \mathbb{N}$, and thus $f$ is harmonic on $T$. From (2) we get

$\begin{array}{cccccc}\left(h_{1}\right) & D_{1}^{k} \Delta f & =q_{2} D_{2}^{k} f & & \\ \left(h_{2}\right) & D_{2}^{k} \Delta f & =q_{3} D_{3}^{k} f-D_{2}^{k} f \\ \left(h_{3}\right) & D_{3}^{k} \Delta f & =p_{3} D_{2}^{k} f+q_{4} D_{4}^{k} f-D_{3}^{k} f \\ \left(h_{4}\right) & D_{4}^{k} \Delta f & =p_{4} D_{3}^{k} f+q_{5} D_{5}^{k} f-D_{4}^{k} f \\ \vdots & \vdots & & & & \\ \left(h_{k-1}\right) & D_{k-1}^{k} \Delta f & =p_{k-1} D_{k-2}^{k} f+q_{k} D_{k}^{k} f-D_{k-1}^{k} f \\ \left(h_{k}\right) & D_{k}^{k} \Delta f & =p_{k} D_{k-1}^{k} f-D_{k}^{k} f .\end{array}$

For $k=1,\left(h_{1}\right)$ yields $D_{1}^{1} \Delta f=0$, since $D_{2}^{1} f=0$. Thus $\Delta f\left(e_{1}\right)=0$, and so $D_{1}^{k} \Delta f=0$ for all $k \in \mathbb{N}$. For $k=2,\left(h_{1}\right)$ becomes $0=q_{2} D_{2}^{2} f$ and $\left(h_{2}\right)$ becomes 
$D_{2}^{2} \Delta f=-D_{2}^{2} f$. Thus $D_{2}^{2} \Delta f=0$, whence $\Delta f\left(e_{2}\right)=0$. So $D_{2}^{k} \Delta f=0$ for all $k \in \mathbb{N}$. Assume inductively that $\Delta f\left(e_{j}\right)=0$ for all $j<k$, so that $D_{j}^{k} \Delta f=0$ for $j<k$. Then the left-hand sides of equations $\left(h_{1}\right)$ through $\left(h_{k-1}\right)$ vanish. Solving these equations starting from the first, we obtain $D_{j}^{k} f=0$ for all $j=1, \ldots, k$. Plugging this into $\left(h_{k}\right)$ yields $D_{k}^{k} \Delta f=p_{k} D_{k-1}^{k} f-D_{k}^{k} f=0$, whence $\Delta f\left(e_{k}\right)=0$, proving the result.

Let us now set up the above procedure for the biharmonic case.

Proof of Theorem 3.1. Let $f^{k}=f^{T_{k}}$, the solution to the Riquier problem on $T_{k}$ with boundary values $f \mid \partial T_{k}$ and $\Delta f \mid \partial T_{k}$. We redefine $D_{j}^{k} f$ to be the difference $f^{k}\left(e_{j}\right)-f\left(e_{j}\right), D_{j}^{k} \Delta f$ to be the difference $\Delta f^{k}\left(e_{j}\right)-\Delta f\left(e_{j}\right)$, and $D_{j}^{k} \Delta^{2} f$ to be the difference $\Delta^{2} f^{k}\left(e_{j}\right)-\Delta^{2} f\left(e_{j}\right)$. Since $f^{k}$ is biharmonic, for all $j \leq k$, $D_{j}^{k} \Delta^{2} f=-\Delta^{2} f\left(e_{j}\right)$.

By the definition of $f^{k}, f$ and $f^{k}$, and similarly, $\Delta f$ and $\Delta f^{k}$, agree on $\left\{u_{j}\right\} \cup$ $\left\{e_{k+1}\right\}$. Thus, we obtain $D_{0}^{k} f=D_{k+1}^{k} f=D_{0}^{k} \Delta f=D_{k+1}^{k} \Delta f=0$ (where we regard $e_{0}$ to be one of the vertices $u_{j}$ ). In addition, we define $D_{-1}^{k} f=D_{k+2}^{k} f=0$.

By a repeated application of (2) we obtain

$$
\begin{aligned}
D_{j}^{k} \Delta^{2} f= & p_{j} D_{j-1}^{k} \Delta f+q_{j+1} D_{j+1}^{k} \Delta f-D_{j}^{k} \Delta f \\
= & p_{j}\left(p_{j-1} D_{j-2}^{k} f+q_{j} D_{j}^{k} f-D_{j-1}^{k} f\right) \\
& \quad+q_{j+1}\left(p_{j+1} D_{j}^{k} f+q_{j+2} D_{j+2}^{k} f-D_{j+1}^{k} f\right) \\
& \quad-\left(p_{j} D_{j-1}^{k} f+q_{j+1} D_{j+1}^{k} f-D_{j}^{k} f\right) \\
= & p_{j-1} p_{j} D_{j-2}^{k} f-2 p_{j} D_{j-1}^{k} f+\left(p_{j} q_{j}+p_{j+1} q_{j+1}+1\right) D_{j}^{k} f \\
& \quad-2 q_{j+1} D_{j+1}^{k} f+q_{j+1} q_{j+2} D_{j+2}^{k} f, \quad \text { for } 1 \leq j \leq k-1 .
\end{aligned}
$$

Since $D_{k+1}^{k} \Delta f=D_{k+1}^{k} f=0$, for $j=k$ we obtain

$$
\begin{aligned}
D_{k}^{k} \Delta^{2} f= & p_{k} D_{k-1}^{k} \Delta f+q_{k+1} D_{k+1}^{k} \Delta f-D_{k}^{k} \Delta f \\
= & p_{k}\left(p_{k-1} D_{k-2}^{k} f+q_{k} D_{k}^{k} f-D_{k-1}^{k} f\right) \\
& \quad-\left(p_{k} D_{k-1}^{k} f+q_{k+1} D_{k+1}^{k} f-D_{k}^{k} f\right) \\
= & p_{k-1} p_{k} D_{k-2}^{k} f-2 p_{k} D_{k-1}^{k} f+\left(p_{k} q_{k}+1\right) D_{k}^{k} f .
\end{aligned}
$$

The hypothesis of Theorem 3.1 is that $D_{1}^{k} f=0$ for all $k \in \mathbb{N}$. Thus we have

$$
\begin{aligned}
& \left(b_{1}\right) \quad D_{1}^{k} \Delta^{2} f=-2 q_{2} D_{2}^{k} f+q_{2} q_{3} D_{3}^{k} f \\
& \left(b_{2}\right) \quad D_{2}^{k} \Delta^{2} f=\left(p_{2} q_{2}+p_{3} q_{3}+1\right) D_{2}^{k} f-2 q_{3} D_{3}^{k} f+q_{3} q_{4} D_{4}^{k} f \\
& \left(b_{3}\right) \quad D_{3}^{k} \Delta^{2} f=-2 p_{3} D_{2}^{k} f+\left(p_{3} q_{3}+p_{4} q_{4}+1\right) D_{3}^{k} f-2 q_{4} D_{4}^{k} f+q_{4} q_{5} D_{5}^{k} f
\end{aligned}
$$




$$
\begin{aligned}
& \left(b_{4}\right) \quad D_{4}^{k} \Delta^{2} f=p_{3} p_{4} D_{2}^{k} f-2 p_{4} D_{3}^{k} f+\left(p_{4} q_{4}+p_{5} q_{5}+1\right) D_{4}^{k} f-2 q_{5} D_{5}^{k} f \\
& +q_{5} q_{6} D_{6}^{k} f \\
& \vdots \quad: \\
& \left(b_{k-1}\right) D_{k-1}^{k} \Delta^{2} f=p_{k-2} p_{k-1} D_{k-3}^{k} f-2 p_{k-1} D_{k-2}^{k} f \\
& +\left(p_{k-1} q_{k-1}+p_{k} q_{k}+1\right) D_{k-1}^{k} f-2 q_{k} D_{k}^{k} f \\
& \left(b_{k}\right) \quad D_{k}^{k} \Delta^{2} f=p_{k-1} p_{k} D_{k-2}^{k} f-2 p_{k} D_{k-1}^{k} f+\left(p_{k} q_{k}+1\right) D_{k}^{k} f \text {. }
\end{aligned}
$$

For $k=1,\left(b_{1}\right)$ yields $D_{1}^{1} \Delta^{2} f=0$, so that $\Delta^{2} f\left(e_{1}\right)=0$, and so $D_{1}^{k} \Delta^{2} f=0$ for all $k \in \mathbb{N}$. For $k=2,\left(b_{1}\right)$ becomes $0=-2 q_{2} D_{2}^{2} f$ and $\left(b_{2}\right)$ becomes $D_{2}^{2} \Delta^{2} f=$ $\left(p_{2} q_{2}+1\right) D_{2}^{2} f$. Thus $D_{2}^{2} \Delta^{2} f=0$, whence $\Delta^{2} f\left(e_{2}\right)=0$ and so $D_{2}^{k} \Delta^{2} f=0$ for all $k \in \mathbb{N}$. For $k=3$, equations $\left(b_{1}\right),\left(b_{2}\right),\left(b_{3}\right)$ yield

$$
\begin{aligned}
0 & =-2 q_{2} D_{2}^{3} f+q_{2} q_{3} D_{3}^{3} f \\
0 & =\left(p_{2} q_{2}+p_{3} q_{3}+1\right) D_{2}^{3} f-2 q_{3} D_{3}^{3} f \\
D_{3}^{3} \Delta^{2} f & =-2 p_{3} D_{2}^{3} f+\left(p_{3} q_{3}+1\right) D_{3}^{3} f .
\end{aligned}
$$

But the determinant of the coefficients of $D_{2}^{3} f$ and $D_{3}^{3} f$ in the top two equations is $q_{2} q_{3}\left(3-p_{2} q_{2}-p_{3} q_{3}\right)>0$. So $D_{2}^{3} f=D_{3}^{3} f=0$, and thus $D_{3}^{3} \Delta^{2} f=0$.

Thus the general problem is to show that the determinant of the coefficients of $D_{2}^{k} f, D_{3}^{k} f, \ldots, D_{k}^{k} f$ in $\left(b_{1}\right), \ldots,\left(b_{k-1}\right)$ is nonzero, whence by substituting into equation $\left(b_{k}\right)$, we get $D_{k}^{k} \Delta^{2} f=0$. Thus $\Delta^{2} f\left(e_{k}\right)=0$, and hence $D_{k}^{l} \Delta^{2} f=0$ for all $l \in \mathbb{N}$, showing by induction that $f$ is biharmonic on $T$.

For all $k \in \mathbb{N}, k \geq 2$, consider the linear system consisting of the equations $\left(b_{1}\right), \ldots,\left(b_{k-1}\right)$ in the variables $D_{2}^{k} f, D_{3}^{k} f, \ldots, D_{k}^{k} f$, and let $A_{k-1}$ be the determinant of its $(k-1) \times(k-1)$ coefficient matrix. The proof will be complete if we show that $A_{k} \neq 0$ for all $k \geq 1$.

We claim that $\left\{A_{k}\right\}_{k=1}^{\infty}$ is a sequence of polynomials in the variables $p_{2}, q_{2}, p_{3}$, $q_{3}, \ldots, p_{k+1}, q_{k+1}$ satisfying the recurrence relation

$$
\begin{aligned}
A_{k+1}= & -2 q_{k+2} A_{k}-q_{k+1} q_{k+2}\left(p_{k+1} q_{k+1}+p_{k+2} q_{k+2}+1\right) A_{k-1} \\
& -2 q_{k} p_{k+1} q_{k+1}^{2} q_{k+2} A_{k-2}-q_{k-1} p_{k} q_{k}^{2} p_{k+1} q_{k+1}^{2} q_{k+2} A_{k-3},
\end{aligned}
$$

for $k \geq 4$, with the initial conditions

$$
\begin{aligned}
A_{1}= & -2 q_{2}, \\
A_{2}= & q_{2} q_{3}\left(3-p_{2} q_{2}-p_{3} q_{3}\right), \\
A_{3}= & q_{2} q_{3} q_{4}\left[-4+2\left(p_{2} q_{2}+p_{3} q_{3}+p_{4} q_{4}\right)\right], \quad \text { and } \\
A_{4}= & q_{2} q_{3} q_{4} q_{5}\left[5-3\left(p_{2} q_{2}+p_{3} q_{3}+p_{4} q_{4}+p_{5} q_{5}\right)\right. \\
& \left.+p_{2} q_{2} p_{4} q_{4}+p_{2} q_{2} p_{5} q_{5}+p_{3} q_{3} p_{5} q_{5}\right] .
\end{aligned}
$$


Since the proof is analogous, for simplicity we verify the recurrence relation only in the homogeneous case, when all values of $p_{j}, q_{j}$ are equal to $p=\frac{1}{q+1}$.

LEMma 3.1. The sequence $\left\{A_{k}(p)\right\}$ of polynomials in p satisfies the recurrence relation

$$
A_{k+1}(p)=-2 p A_{k}(p)-\left(2 p^{4}+p^{2}\right) A_{k-1}(p)-2 p^{5} A_{k-2}(p)-p^{8} A_{k-3}(p),
$$

for all $k \geq 4$, with the initial conditions $A_{1}(p)=-2 p, A_{2}(p)=3 p^{2}-2 p^{4}, A_{3}(p)=$ $-4 p^{3}+6 p^{5}$, and $A_{4}(p)=5 p^{4}-12 p^{6}+3 p^{8}$.

Proof. First observe that $A_{k}$ is the determinant of the $k \times k$ matrix for which the main diagonal, the diagonal above it, and the three diagonals below it consist of identical entries, $-2 p, p^{2}, 2 p^{2}+1,-2 p$, and $p^{2}$, respectively, and all other entries are 0 :

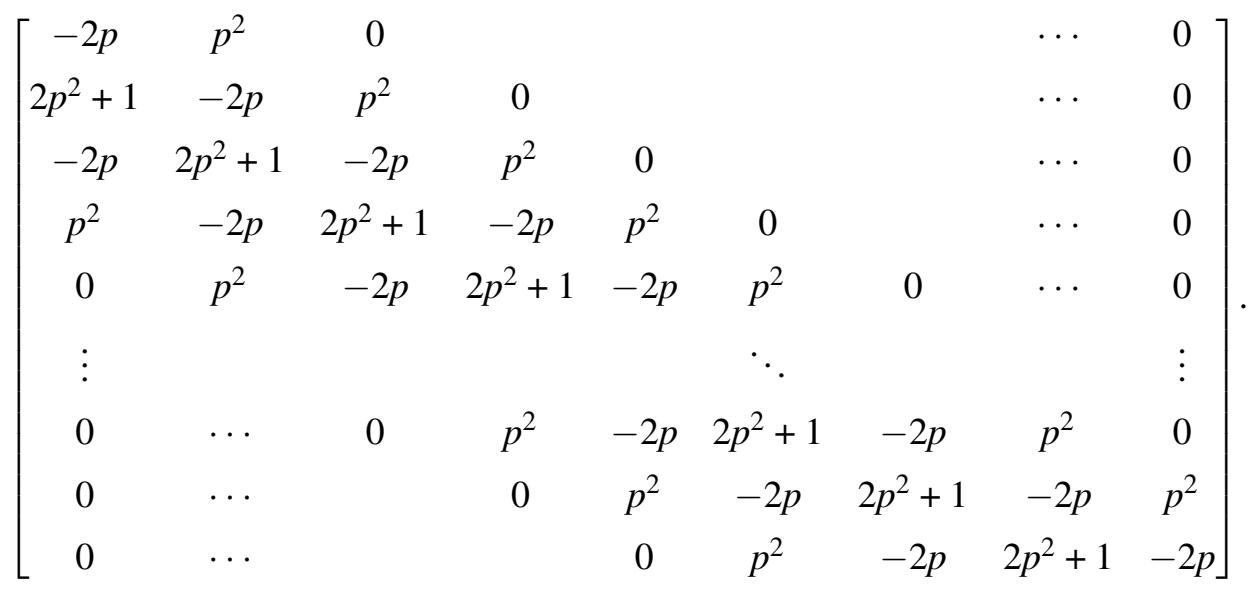

This type of matrix is known as a Toeplitz matrix. It is straightforward to compute the values of $A_{k}$ for $k=1,2,3,4$. Expanding the determinant across the last column, we get $A_{k+1}=-2 p A_{k}-p^{2} F_{k}$, where

$$
F_{k}=\left|\begin{array}{cccccccc}
-2 p & p^{2} & 0 & & & & \cdots & 0 \\
2 p^{2}+1 & -2 p & p^{2} & 0 & & & \cdots & 0 \\
-2 p & 2 p^{2}+1 & -2 p & p^{2} & 0 & & \cdots & 0 \\
p^{2} & -2 p & 2 p^{2}+1 & -2 p & p^{2} & 0 & \cdots & 0 \\
\vdots & & & & \ddots & & & \vdots \\
0 & \ldots & 0 & p^{2} & -2 p & 2 p^{2}+1 & -2 p & p^{2} \\
0 & \ldots & & & 0 & p^{2} & -2 p & 2 p^{2}+1
\end{array}\right| .
$$


Expanding this determinant across the last column we obtain $F_{k}=\left(2 p^{2}+1\right)$ $A_{k-1}-p^{2} G_{k-1}$, where

$$
G_{k-1}=\left|\begin{array}{cccccccc}
-2 p & p^{2} & 0 & & & & \cdots & 0 \\
2 p^{2}+1 & -2 p & p^{2} & 0 & & & \cdots & 0 \\
-2 p & 2 p^{2}+1 & -2 p & p^{2} & 0 & & \cdots & 0 \\
p^{2} & -2 p & 2 p^{2}+1 & -2 p & p^{2} & 0 & \cdots & 0 \\
\vdots & & & & \ddots & & & \vdots \\
0 & \ldots & 0 & p^{2} & -2 p & 2 p^{2}+1 & -2 p & p^{2} \\
0 & \cdots & & & 0 & 0 & p^{2} & -2 p
\end{array}\right| .
$$

Repeating the expansion across the last column two more times we see that $G_{k-1}=-2 p A_{k-2}-p^{4} A_{k-3}$, which, together with the above relations, yields the result.

Observe that the recurrence relation (3) can be expressed entirely in terms of the products $p_{j} q_{j}$ by defining

$$
B_{k}=(-1)^{k} \frac{A_{k}}{\prod_{j=2}^{k+1} q_{j}}
$$

yielding

$$
\begin{aligned}
B_{k+1}= & 2 B_{k}-\left(p_{k+1} q_{k+1}+p_{k+2} q_{k+2}+1\right) B_{k-1} \\
& +2 p_{k+1} q_{k+1} B_{k-2}-p_{k} q_{k} p_{k+1} q_{k+1} B_{k-3},
\end{aligned}
$$

for $k \geq 4$, with the initial conditions

$$
\begin{aligned}
& B_{1}=2, \quad B_{2}=3-p_{2} q_{2}-p_{3} q_{3}, \quad B_{3}=4-2\left(p_{2} q_{2}+p_{3} q_{3}+p_{4} q_{4}\right) \\
& B_{4}=5-3\left(p_{2} q_{2}+p_{3} q_{3}+p_{4} q_{4}+p_{5} q_{5}\right)+p_{2} q_{2} p_{4} q_{4}+p_{2} q_{2} p_{5} q_{5}+p_{3} q_{3} p_{5} q_{5}
\end{aligned}
$$

Note that since for all $j, 0<p_{j}, q_{j}<1$, we have $p_{j} q_{j}+p_{j+1} q_{j+1}<p_{j}+q_{j+1} \leq 1$. So, for example, one sees immediately that $B_{2}$ and $B_{3}$ are always positive.

In Theorem 3.2 we shall prove that $B_{k}>0$ for all $k \in \mathbb{N}$, and thus each $A_{k} \neq 0$. This completes the proof of Theorem 3.1.

We have postponed Theorem 3.2 because it is long and requires the use of two lemmas. 
For all $j \in \mathbb{N}$, let $p_{j}, q_{j} \in[0,1]$ be such that $p_{j}+q_{j+1} \leq 1$, and set $s_{j}=p_{j} q_{j}$. Let $s=\left(s_{1}, s_{2}, \ldots\right)$. Define a sequence $\left\{B_{n}(s)\right\}_{n=-\infty}^{\infty}$ of polynomials in the variables $s_{1}, s_{2}, \ldots$ as follows:

$$
B_{n}(s)= \begin{cases}0 & \text { if } n<0 \\ 1 & \text { if } n=0\end{cases}
$$

and for $n>0$

$$
\begin{aligned}
B_{n}(s)= & 2 B_{n-1}(s)-\left(s_{n-1}+s_{n}+1\right) B_{n-2}(s) \\
& +2 s_{n-1} B_{n-3}(s)-s_{n-2} s_{n-1} B_{n-4}(s) .
\end{aligned}
$$

Thus $B_{1}(s)=2, B_{2}(s)=3-\left(s_{1}+s_{2}\right), B_{3}(s)=4-2\left(s_{1}+s_{2}+s_{3}\right)$, and $B_{4}(s)=$ $5-3\left(s_{1}+s_{2}+s_{3}+s_{4}\right)+s_{1} s_{3}+s_{1} s_{4}+s_{2} s_{4}$. Notice that $B_{n}(s)$ depends only on $s_{1}, \ldots, s_{n}$.

Definition 3.1. We shall call $s$ an interior point if all values of $p_{j}$ and $q_{j}$ are in the interval $(0,1)$, a boundary point, otherwise.

Warning. Notice that our definition of interior point does not require $p_{j}+q_{j+1}<$ 1. Also, for algebraic convenience the indices have been reindexed from the way they were used in Theorem 3.1, as can be seen by comparing $B_{1}(s), \ldots, B_{4}(s)$ just defined to $B_{1}, \ldots, B_{4}$ in (5).

THEOREM 3.2. For all $s=\left(s_{1}, s_{2}, \ldots\right)$ and for all integers $n, B_{n}(s) \geq 0$, and $B_{n}(s)>0$ if $s$ is an interior point and $n$ is nonnegative.

For the proof we need the following notation and two lemmas.

Notation 3.1. For $n \geq 3$ and $s=\left(s_{1}, \ldots, s_{n}\right)$, let

$$
s^{(k)}=\left(s_{1}, \ldots, s_{k-2}, 0, s_{k+2}, \ldots, s_{n}\right),
$$

if $n \geq 5$ and $k \in\{3, \ldots, n-2\}$,

$$
\begin{aligned}
s^{(1)} & =\left(s_{3}, \ldots, s_{n}\right), \\
s^{(2)} & = \begin{cases}0 & \text { if } n=3 \\
\left(0, s_{4}, \ldots, s_{n}\right) & \text { if } n \geq 4,\end{cases} \\
s^{(n-1)} & = \begin{cases}0 & \text { if } n=3 \\
\left(s_{1}, \ldots, s_{n-3}, 0\right) & \text { if } n \geq 4,\end{cases} \\
s^{(n)} & =\left(s_{1}, \ldots, s_{n-2}\right) .
\end{aligned}
$$

For $n \leq 2$, let $s^{(k)}=0$ for any integer $k$. 
LEMmA 3.2. For all $k \in\{1, \ldots, n\}, \frac{\partial B_{n}(s)}{\partial s_{k}}=-B_{n-2}\left(s^{(k)}\right)$.

Proof. It is easy to check that the result holds for $n=1,2,3$. Assume $n \geq 4$ and that

$$
\frac{\partial B_{j}(s)}{\partial s_{k}}=-B_{j-2}\left(s^{(k)}\right) \text { for all } j \in\{1, \ldots, n-1\} \text { and } k \in\{1, \ldots, j\} .
$$

Then, using (6), we obtain $\frac{\partial B_{n}(s)}{\partial s_{n}}=-B_{n-2}(s)=-B_{n-2}\left(s^{(n)}\right)$, because none of the other terms in the right-hand side of the recurrence relation depends on $s_{n}$. Next, note that using (6) and the induction hypothesis, we have

$$
\begin{aligned}
\frac{\partial B_{n}(s)}{\partial s_{n-1}} & =2 \frac{\partial B_{n-1}(s)}{\partial s_{n-1}}-B_{n-2}(s)+2 B_{n-3}(s)-s_{n-2} B_{n-4}(s) \\
& =-B_{n-2}(s)-s_{n-2} B_{n-4}(s)
\end{aligned}
$$

On the other hand, by our inductive hypothesis, we have

$$
\frac{\partial}{\partial s_{n-2}}\left(B_{n-2}(s)+s_{n-2} B_{n-4}(s)\right)=-B_{n-4}(s)+B_{n-4}(s)=0 .
$$

Thus $B_{n-2}(s)+s_{n-2} B_{n-4}(s)$ is independent of $s_{n-2}$ and so, after replacing $s_{n-2}$ by 0 , we get

$$
B_{n-2}(s)+s_{n-2} B_{n-4}(s)=B_{n-2}\left(s^{(n-1)}\right) .
$$

Hence $\frac{\partial B_{n}(s)}{\partial s_{n-1}}=-B_{n-2}\left(s^{(n-1)}\right)$.

Next, again using (6), we obtain

$$
\begin{aligned}
\frac{\partial B_{n}(s)}{\partial s_{n-2}} & =2 \frac{\partial B_{n-1}(s)}{\partial s_{n-2}}-\left(s_{n-1}+s_{n}+1\right) \frac{\partial B_{n-2}(s)}{\partial s_{n-2}}-s_{n-1} B_{n-4}(s) \\
& =-2 B_{n-3}\left(s^{(n-2)}\right)+\left(s_{n-1}+s_{n}+1\right) B_{n-4}\left(s^{(n-2)}\right)-s_{n-1} B_{n-4}(s) \\
& =-2 B_{n-3}\left(s^{(n-2)}\right)+\left(s_{n}+1\right) B_{n-4}(s),
\end{aligned}
$$

since $B_{n-4}\left(s^{(n-2)}\right)=B_{n-4}(s)$. On the other hand, using (6), we have

$$
\begin{aligned}
B_{n-2}\left(s^{(n-2)}\right) & =B_{n-2}\left(s_{1}, \ldots, s_{n-4}, 0, s_{n}\right) \\
& =2 B_{n-3}\left(s_{1}, \ldots, s_{n-4}, 0\right)-\left(s_{n}+1\right) B_{n-4}\left(s_{1}, \ldots, s_{n-4}\right) \\
& =2 B_{n-3}\left(s^{(n-2)}\right)-\left(s_{n}+1\right) B_{n-4}(s)
\end{aligned}
$$

proving that $\frac{\partial B_{n}(s)}{\partial s_{n-2}}=-B_{n-2}\left(s^{(n-2)}\right)$. 
For $k<n-2$, using (6) and the inductive hypothesis, we have

$$
\begin{aligned}
\frac{\partial B_{n}(s)}{\partial s_{k}}= & -2 B_{n-3}\left(s^{(k)}\right)+\left(s_{n-1}+s_{n}+1\right) B_{n-4}\left(s^{(k)}\right) \\
& -2 s_{n-1} B_{n-5}\left(s^{(k)}\right)+s_{n-2} s_{n-1} B_{n-6}\left(s^{(k)}\right) .
\end{aligned}
$$

Also,

$$
\begin{aligned}
B_{n-2}\left(s^{(k)}\right)= & B_{n-2}\left(s_{1}, \ldots, s_{k-2}, 0, s_{k+2}, \ldots, s_{n}\right) \\
= & 2 B_{n-3}\left(s^{(k)}\right)-\left(s_{n-1}+s_{n}+1\right) B_{n-4}\left(s^{(k)}\right) \\
& +2 s_{n-1} B_{n-5}\left(s^{(k)}\right)-s_{n-2} s_{n-1} B_{n-6}\left(s^{(k)}\right),
\end{aligned}
$$

which, together with (7), yields $\frac{\partial B_{n}(s)}{\partial s_{k}}=-B_{n-2}\left(s^{(k)}\right)$. This completes the proof.

Definition 3.2. We will call $s=\left(s_{1}, \ldots, s_{n}\right)$ a vertex if each $s_{j}$ is 0 or 1 .

Observe that $s_{j}=1$ implies $p_{j}=1$, so that $q_{j+1}=0$, whence $s_{j+1}=0$.

Notation 3.2. For a vertex $s=\left(s_{1}, \ldots, s_{n}\right)$, set $|s|=\sum_{j=0}^{n} s_{j} \in\{0,1, \ldots, n\}$.

LEMMA 3.3. If $s=\left(s_{1}, \ldots, s_{n}\right)$ is a vertex, then

$$
B_{n}(s)= \begin{cases}n+1 & \text { if }|s|=0 \\ 2 & \text { if }|s|=1 \\ 0 & \text { if }|s| \geq 2\end{cases}
$$

Proof. By inspection, we see that the result is true for $n=1,2,3,4$. So assume the formula for $B_{k}(s)$ holds for all $k<n$, where $n \geq 5$. We now compute $B_{n}(s)$ for a vertex $s$.

Case 1. $|s|=0$. Then

$$
B_{n}(s)=2 B_{n-1}(s)-B_{n-2}(s)=2 n-(n-1)=n+1 .
$$

Case 2. $|s|=1$. If $s_{n}=1$, then $s_{j}=0$ for all $j<n$. Thus

$$
B_{n}(s)=2 B_{n-1}(s)-2 B_{n-2}(s)=2 n-2(n-1)=2 .
$$

If $s_{n-1}=1$, then $s_{j}=0$ for $j \neq n-1$. So

$$
B_{n}(s)=2 B_{n-1}(s)-2 B_{n-2}(s)+2 B_{n-3}(s)=4-2(n-1)+2(n-2)=2 .
$$


If $s_{j}=1$ for some $j<n-1$, then

$$
B_{n}(s)=2 B_{n-1}(s)-B_{n-2}(s)=4-2=2 .
$$

Case 3. $|s|=2$. If $s_{n}=1$, then $s_{n-1}$ must be 0 , by definition of vertex. So

$$
B_{n}(s)=2 B_{n-1}(s)-2 B_{n-2}(s)=4-4=0 .
$$

If $s_{n-1}=1$ and $s_{n}=s_{n-2}=0$, then

$$
B_{n}(s)=2 B_{n-1}(s)-2 B_{n-2}(s)+2 B_{n-3}(s)=0-4+4=0 .
$$

If $s_{n}=s_{n-1}=0$, then by induction $B_{n-1}(s)=B_{n-2}(s)=0$, and thus $B_{n}(s)=0$.

Case 4. $|s|>2$. Then $\sum_{j=1}^{n-2} s_{j} \geq 2$, so that $B_{n-1}(s)=B_{n-2}(s)=0$. Hence

$$
B_{n}(s)=2 s_{n-1} B_{n-3}(s)-s_{n-2} s_{n-1} B_{n-4}(s) .
$$

If $s_{n-1}=0$, then (8) implies that $B_{n}(s)=0$. If $s_{n-1}=1$, then by definition of vertex, $s_{n}=s_{n-2}=0$ and so $\sum_{j=1}^{n-3} s_{j} \geq 2$. Thus $B_{n-3}(s)=0$, by induction. Again, (8) implies that $B_{n}(s)=0$, completing the proof.

Proof of Theorem 3.2. From the initial conditions defining $B_{n}$, it is clear that for $n=0,1, B_{n}(s)>0$. Arguing by induction on $n$, let us assume $B_{k}(s) \geq 0$ for all $k<n$, where $n \geq 2$. Then by Lemma 3.2, $\frac{\partial B_{n}(s)}{\partial s_{j}} \leq 0$, for all $j \leq n$. Thus, the minimum value of $B_{n}(s)$ will occur when $s_{j}=p_{j} q_{j}$ is as large as possible. Since $q_{j} \leq 1-p_{j-1}$, we can assume that $q_{j}=1-p_{j-1}$. So from now on we take $s_{j}=p_{j}\left(1-p_{j-1}\right)$. Since the only $s_{i}$ that involve $p_{j}$ are $s_{j}$ and $s_{j+1}$, we get

$$
\begin{aligned}
\frac{\partial B_{n}(s)}{\partial p_{j}} & =\frac{\partial B_{n}(s)}{\partial s_{j}} \frac{\partial s_{j}}{\partial p_{j}}+\frac{\partial B_{n}(s)}{\partial s_{j+1}} \frac{\partial s_{j+1}}{\partial p_{j}} \\
& =-B_{n-2}\left(s^{(j)}\right)\left(1-p_{j-1}\right)+B_{n-2}\left(s^{(j+1)}\right) p_{j+1}
\end{aligned}
$$

by Lemma 3.2. Since $s^{(j)}$ and $s^{(j+1)}$ do not depend on $s_{j}$, it follows that $\frac{\partial B_{n}(s)}{\partial p_{j}}$ is constant with respect to $p_{j}$. Thus, $B_{n}(s)$ reaches its minimum at $p_{j}=0$ or $p_{j}=1$. By our assumption that $s_{j}=p_{j}\left(1-p_{j-1}\right)$, we deduce that the corresponding point $s=\left(s_{1}, \ldots, s_{n}\right)$ is a vertex. Hence, $B_{n}(s)$ reaches its minimum at vertices $s$. By Lemma 3.3, we obtain $B_{n}(s) \geq 0$.

We now need to prove that if $s$ is an interior point, then $B_{n}(s)>0$. Assume by induction that $B_{k}(s)>0$ for $k<n$, where $n \geq 2$. Since by Lemma 3.2 and the inductive hypothesis $\frac{\partial B_{n}(s)}{\partial s_{n}}=-B_{n-2}\left(s^{(n)}\right)=-B_{n-2}\left(s_{1}, \ldots, s_{n-2}\right)<0$, 
the function $B_{n}$ is strictly decreasing in the last variable. If $B_{n}(s)$ were 0 , then $B_{n}\left(s_{1}, \ldots, s_{n-1}, t_{n}\right)<0$, for any $t_{n}>s_{n}$, and in particular, for some other interior point, yielding a contradiction. Thus, $B_{n}(s)>0$, completing the proof.

Remark 3.1. The analogue of Theorem 3.1 for triharmonic functions also holds. We were able to follow the analogous procedure developed in this section to arrive at the following sixth order linear homogeneous recurrence relation

$$
\begin{aligned}
B_{k+1}= & 3 B_{k}-\left(p_{k} q_{k}+p_{k+1} q_{k+1}+p_{k+2} q_{k+2}+3\right) B_{k-1} \\
& +\left(3 p_{k} q_{k}+3 p_{k+1} q_{k+1}+1\right) B_{k-2} \\
& -p_{k} q_{k}\left(p_{k-1} q_{k-1}+p_{k} q_{k}+p_{k+1} q_{k+1}+3\right) B_{k-3} \\
& +3 p_{k-1} q_{k-1} p_{k} q_{k} B_{k-4}-p_{k-2} q_{k-2} p_{k-1} q_{k-1} p_{k} q_{k} B_{k-5},
\end{aligned}
$$

where

$$
B_{k}=(-1)^{k} \frac{A_{k}}{\prod_{j=1}^{k} q_{j} q_{j+1}},
$$

having denoted by $A_{k}$ the determinant of the matrix consisting of the coefficients of $D_{j}^{l} f(1 \leq j, l \leq k)$. It turns out that Lemma 3.2 also holds for this sequence $\left\{B_{n}\right\}$, and hence we can use an inductive argument to show that $B_{n}>0$ for all positive integers $n$. We omit the proofs of these results, since the methods are analogous to those developed for the biharmonic case, but the complexity of computations is much greater. We suspect that the Globevnik-Rudin characterization of $n$-polyharmonic functions holds for any order $n$.

\section{Characterization of polyharmonic functions on a homogeneous tree.} The problem of characterizing polyharmonic functions on a domain in $\mathbb{R}^{d}$ was studied by Almansi in [2]. He proved the following result.

TheOREM. (Almansi) If $f$ is polyharmonic of order $n$ in a domain $D \subset \mathbb{R}^{d}$ which is starlike with respect to 0 , then there exist unique harmonic functions $h_{0}, \ldots, h_{n-1}$ on $D$, such that

$$
f(x)=\sum_{k=0}^{n-1}\|x\|^{2 k} h_{k}(x) \text { for all } x \in D .
$$

For a proof see [2] or [4], p. 4.

In this section we give an integral representation of polyharmonic functions in the spirit of Almansi's Theorem in the case of a homogeneous tree.

Notation 4.1. Fix a vertex $e$ as the root of a tree $T$ and use the notations $k_{\omega}(v)$ and $P_{\omega}(v)$ for $k_{\omega}(e, v)$ and $P_{\omega}(e, v)$, respectively. Let $<$ be the partial ordering 
on $T \cup \Omega$ given by $v<u$ if $v \in\left[e, u^{-}\right]$or $v<\omega$ if $\omega \in I_{v}$, where

$$
I_{v}=I_{v}^{e}=\{\omega \in \Omega: v \in[e, \omega)\} .
$$

Then for $v \in T$ and $\lambda \in T \cup \Omega$, let $v \wedge \lambda$ be $\inf \{v, \lambda\}$, the vertex $u$ of largest length such that $u \leq v$ and $u \leq \lambda$. Observe that for all $\omega \in \Omega, k_{\omega}(v)=2|v \wedge \omega|-|v|$.

Definition 4.1. Let $T$ be a homogeneous tree of degree $q+1$. Let $\mu_{n}(n$ nonnegative integer) be the operator that averages the values of the function at the vertices at distance $n$. Thus, if $f$ is a function on $T$, then

$$
\mu_{n} f(v)=\frac{1}{c_{n}} \sum_{d(w, v)=n} f(w) \quad \text { where } c_{n}= \begin{cases}1 & \text { if } n=0 \\ (q+1) q^{n-1} & \text { if } n \geq 1\end{cases}
$$

So, for example, $\Delta=\mu_{1}-1_{T}$, where $1_{T}$ is the identity operator.

LEMMA 4.1. For a fixed $\omega \in \Omega$, we have

(a) $\Delta k_{\omega}$ is the constant $\frac{1-q}{q+1}$;

(b) For all $m \in \mathbb{N}$ there exist constants $a_{0}^{(m)}, \ldots, a_{m-1}^{(m)}$ such that

$$
\Delta\left(k_{\omega}^{m} P_{\omega}\right)=\sum_{j=0}^{m-1} a_{j}^{(m)} k_{\omega}^{j} P_{\omega}
$$

Consequently, $k_{\omega}$ and $k_{\omega}^{m} P_{\omega}$ are polyharmonic functions. In fact, $\Delta^{m+1} k_{\omega}^{m} P_{\omega}=0$.

Proof. Let $v \in T$ and set $k_{\omega}(v)=n \in \mathbb{Z}$. Then

$$
\Delta k_{\omega}(v)=\mu_{1} k_{\omega}(v)-k_{\omega}(v)=\frac{q(n-1)+n+1}{q+1}-n=\frac{1-q}{q+1},
$$

proving (a). Moreover

$$
\begin{aligned}
\Delta\left(k_{\omega}^{m} P_{\omega}\right)(v) & =\mu_{1}\left(k_{\omega}^{m} P_{\omega}\right)(v)-k_{\omega}(v)^{m} P_{\omega}(v) \\
& =\frac{q(n-1)^{m} q^{n-1}+(n+1)^{m} q^{n+1}}{q+1}-n^{m} q^{n} \\
& =\frac{q^{n}}{q+1}\left[(n-1)^{m}+(n+1)^{m} q-n^{m}(q+1)\right] \\
& =\frac{q^{n}}{q+1} \sum_{j=0}^{m-1}\left\{\left(\begin{array}{c}
m \\
j
\end{array}\right)\left[(-1)^{m-j}+q\right] n^{j}\right\} \\
& =\sum_{j=0}^{m-1}\left(\begin{array}{c}
m \\
j
\end{array}\right)\left[\frac{(-1)^{m-j}+q}{q+1}\right] k_{\omega}^{j}(v) P_{\omega}(v),
\end{aligned}
$$


completing the proof of (b). From (a) it follows that $\Delta^{2} k_{\omega}=0$, so $k_{\omega}$ is biharmonic. For $m=0, \Delta^{m+1} k_{\omega}^{m} P_{\omega}=\Delta P_{\omega}=0$. Let $m>0$ and assume inductively that $\Delta^{j+1} k_{\omega}^{j} P_{\omega}=0$ for $j<m$. Then as a consequence of (b), we see that

$$
\Delta^{m+1}\left(k_{\omega}^{m} P_{\omega}\right)=\sum_{j=0}^{m-1} a_{j}^{(m)} \Delta^{m}\left(k_{\omega}^{j} P_{\omega}\right)=0,
$$

where

$$
a_{m-1}^{(m)}=\left(\begin{array}{c}
m \\
m-1
\end{array}\right)\left(\frac{q-1}{q+1}\right)=m\left(\frac{q-1}{q+1}\right) .
$$

Hence,

$$
\Delta^{m}\left(k_{\omega}^{m} P_{\omega}\right)=m !\left(\frac{q-1}{q+1}\right)^{m} P_{\omega}
$$

Since $P_{\omega}$ is harmonic, $\Delta^{t}\left(k_{\omega}^{m} P_{\omega}\right)=0$ for all $t>m$.

Using a simple inductive argument we deduce:

Lemma 4.2. Fix $\omega \in \Omega$. Then for all nonnegative integers $m$ there exist constants $\alpha_{j, m}$, with $1 \leq j \leq m+1$ such that

$$
k_{\omega}^{m} P_{\omega}=\Delta\left(\sum_{j=1}^{m+1} \alpha_{j, m} k_{\omega}^{j} P_{\omega}\right) .
$$

We are now ready to characterize polyharmonic functions:

THEOREM 4.1. Let $T$ be a homogeneous tree of degree $q+1$. A function $f$ on $T$ is polyharmonic of order $n$ if and only if there exist distributions $\nu_{m}(m=0, \ldots, n-1$, $n \in \mathbb{N}$ ) on $\Omega$ such that

$$
f=\sum_{m=0}^{n-1} \int_{\Omega} k_{\omega}^{m} P_{\omega} d \nu_{m}(\omega)
$$

Furthermore, the representation (10) is unique.

OBSERVATION 4.1. Let $v$ be any vertex of length $N \geq 1$, and label the vertices on the path $[e, v]$ as $v_{0}, \ldots, v_{N}$, where $v_{0}=e, v_{N}=v$. The integrals in $(10)$ can be easily calculated by partitioning $\Omega$ into the sets $I_{v_{N}}, I_{v_{N-1}}-I_{v_{N}}, I_{v_{N-2}}-I_{v_{N-1}}, \ldots, I_{v_{0}}-I_{v_{1}}$, because for $\omega$ in these sets the value of $k_{\omega}(v)$ is $N, N-2, N-4, \ldots,-N$, respectively, and $P_{\omega}=q^{k_{\omega}}$. That is,

$$
k_{\omega}(v)= \begin{cases}2 j-N & \text { for } \omega \in I_{v_{j}}-I_{v_{j+1}}, 0 \leq j \leq N-1, \\ N & \text { for } \omega \in I_{v_{N}} .\end{cases}
$$


Thus, (10) becomes

$$
f(v)=\sum_{m=0}^{n-1}\left\{\sum_{j=0}^{N-1}(2 j-N)^{m} q^{2 j-N} \nu_{m}\left(I_{v_{j}}-I_{v_{j+1}}\right)+N^{m} q^{N} \nu_{m}\left(I_{v_{N}}\right)\right\},
$$

where $v_{N}=v$ and $I_{v_{0}}=\Omega$.

In particular, if $f$ is a biharmonic function, then its integral representation, to be used in Section 5 , is given by

$$
\begin{aligned}
f(v)= & \sum_{j=0}^{N-1} q^{2 j-N} \nu_{0}\left(I_{v_{j}}-I_{v_{j+1}}\right)+q^{N} \nu_{0}\left(I_{v_{N}}\right) \\
& +\sum_{j=0}^{N-1}(2 j-N) q^{2 j-N} \nu_{1}\left(I_{v_{j}}-I_{v_{j+1}}\right)+N q^{N} \nu_{1}\left(I_{v_{N}}\right),
\end{aligned}
$$

for $|v|=N$ with $[e, v]=\left[v_{0}, \ldots, v_{N}\right]$. This reduces to the first two summands if $f$ is harmonic.

Notation 4.2. We denote by $P \nu$ the integral $\int_{\Omega} P_{\omega} d \nu(\omega)$ and by $k P \nu$ the integral $\int_{\Omega} k_{\omega} P_{\omega} d \nu(\omega)$. More generally, if $K$ is a function on $\Omega \times T$ and $\nu$ is a distribution on $\Omega$, we define the function $K \nu$ on $T$ by

$$
K \nu(v)=\int_{\Omega} K(\omega, v) d \nu(\omega)
$$

We proceed with the proof of Theorem 4.1.

Proof. It is clear that the function on the right-hand side of (10) is polyharmonic of order $n$, since

$$
\Delta^{n}\left(\sum_{m=0}^{n-1} \int_{\Omega} k_{\omega}^{m} P_{\omega} d \nu_{m}(\omega)\right)=\sum_{m=0}^{n-1} \int_{\Omega} \Delta^{n}\left(k_{\omega}^{m} P_{\omega}\right) d \nu_{m}(\omega)=0
$$

by Lemma 4.1. Conversely, assume $\Delta^{n} f=0$. We prove the existence of the distributions $\nu_{m}$ satisfying (10) by induction on $n$. For $n=1, f$ is harmonic, so there exists a distribution $\nu_{0}$ on $\Omega$ such that

$$
f=\int_{\Omega} P_{\omega} d \nu_{0}(\omega)
$$

proving the result. Next, assume the integral representation (2) holds for functions $g$ such that $\Delta^{k} g=0$, where $k=1, \ldots, n-1$ for some $n \geq 2$. Then $\Delta^{n-1}(\Delta f)=0$, 
so by induction and Lemma 4.2 we have

$$
\begin{aligned}
\Delta f & =\sum_{m=0}^{n-2} \int_{\Omega} k_{\omega}^{m} P_{\omega} d \nu_{m}(\omega) \\
& =\sum_{m=0}^{n-2} \int_{\Omega} \Delta\left(\sum_{j=1}^{m+1} \alpha_{j, m} k_{\omega}^{j} P_{\omega}\right) d \nu_{m}(\omega) \\
& =\Delta\left(\sum_{j=1}^{n-1} \int_{\Omega} k_{\omega}^{j} P_{\omega} d \rho_{j}(\omega)\right)
\end{aligned}
$$

where the distributions $\rho_{j}$ are defined as

$$
\rho_{j}=\sum_{m=j-1}^{n-2} \alpha_{j, m} \nu_{m}
$$

Hence the function

$$
f-\sum_{j=1}^{n-1} \int_{\Omega} k_{\omega}^{j} P_{\omega} d \rho_{j}(\omega)
$$

is harmonic, and thus it can be represented as $\int_{\Omega} P_{\omega} d \rho_{0}(\omega)$ for some distribution $\rho_{0}$, completing the proof of the existence of the distributions in (10).

To prove uniqueness, we show that the distributions $\nu_{m}$ in (10) can be calculated in terms of any given polyharmonic function $f$. We recall from [13] that a distribution $\nu$ is a finitely additive function on the measurable subsets of $\Omega$, which is determined by its values on the basic measurable sets $I_{v}$, for each $v \in T$.

If $f$ is a harmonic function, so that $f=\int_{\Omega} P_{\omega} d \nu(\omega)$, then the values of $\nu\left(I_{v}\right)$ can be calculated as follows:

$$
\nu\left(I_{v}\right)=\frac{q}{(q-1) c_{|v|}}\left(f(v)-\frac{1}{q} f^{-}(v)\right)
$$

where $f^{-}$is the function defined by $f^{-}(v)=f\left(v^{-}\right)$(cf. [13]).

Thus for a polyharmonic function of order 1 (i.e., a harmonic function), the integral representation (10) is unique.

Next, assume that for any polyharmonic function of order $n$ the representation (10) is uniquely determined in terms of the values of the function, and let

$$
f=\sum_{m=0}^{n} \int_{\Omega} k_{\omega}^{m} P_{\omega} d \nu_{m}(\omega)
$$


be a polyharmonic function of order $n+1$. Using (9), we see that

$$
\Delta^{n} f=n !\left(\frac{q-1}{q+1}\right)^{n} \int_{\Omega} P_{\omega} d \nu_{n}(\omega)
$$

which is harmonic. So the values of $\nu_{n}\left(I_{v}\right)(v \in T)$ can be calculated explicitly as follows:

$$
\nu_{n}\left(I_{v}\right)=\frac{1}{n !}\left(\frac{q+1}{q-1}\right)^{n} \frac{q}{(q-1) c_{|v|}}\left(\Delta^{n} f(v)-\frac{1}{q}\left(\Delta^{n} f\right)^{-}(v)\right) .
$$

Since

$$
\Delta^{n}\left(f-\int_{\Omega} k_{\omega}^{n} P_{\omega} d \nu_{n}(\omega)\right)=\Delta^{n} f-\int_{\Omega} \Delta^{n}\left(k_{\omega}^{n} P_{\omega}\right) d \nu_{n}(\omega)=0
$$

the function

$$
f-\int_{\Omega} k_{\omega}^{n} P_{\omega} d \nu_{n}(\omega)=\sum_{m=0}^{n-1} \int_{\Omega} k_{\omega}^{m} P_{\omega} d \nu_{m}(\omega)
$$

is polyharmonic of order $n$, so by the inductive hypothesis, the remaining distributions $\nu_{m}(0 \leq m \leq n-1)$ are uniquely determined.

5. Polymartingales. In this section, we introduce and study polymartingales on a homogeneous tree.

Definition 5.1. A function $\varphi$ on a homogeneous tree $T$ of degree $q+1$ is a martingale if it satisfies the condition that the average value of $\varphi$ on the children of any vertex $v$ is equal to its value at $v$, that is, letting

$$
M \varphi(v)= \begin{cases}\frac{1}{q} \sum_{u^{-}=v} \varphi(u)-\varphi(v) & \text { if } v \neq e \\ \frac{1}{q+1} \sum_{u \sim e} \varphi(u)-\varphi(e) & \text { if } v=e,\end{cases}
$$

then $\varphi$ is a martingale if and only if $M \varphi=0$.

We recall from [27] (see $\S 3$ ), that there are one-to-one correspondences among the space of harmonic functions $\{f\}$ on a homogeneous tree $T$, the space of martingales $\{\varphi\}$ on $T$ and the space of distributions $\{\nu\}$ on the boundary of $T$, given by

$$
\varphi(v)= \begin{cases}\frac{q}{q-1}\left(f(v)-\frac{1}{q} f\left(v^{-}\right)\right), & \text {for } v \neq e \\ f(e) & \text { for } v=e\end{cases}
$$




$$
\begin{aligned}
f(v) & = \begin{cases}\frac{q-1}{q} \sum_{j=1}^{|v|} q^{j-|v|} \varphi\left(v_{j}\right)+q^{-|v|} \varphi(e) & \text { for } v \neq e \\
\varphi(e) & \text { for } v=e\end{cases} \\
\nu\left(I_{v}\right) & =\frac{\varphi(v)}{c_{|v|}}, \\
f & =P \nu,
\end{aligned}
$$

where $v_{j}(j=1, \ldots,|v|)$ is the ancestor of $v$ of length $j$. We recall that $c_{0}=1$ and $c_{n}=(q+1) q^{n-1}$ for $n \geq 1$.

Notice that formula (12) is the same as the above without explicit reference to $\varphi$. In this section, we introduce the concept of polymartingale of order $n$ by replacing $M$ with $M^{n}$, for $n \geq 2$, and give 1-1 correspondences among the space of $n$-polyharmonic functions $\{f\}$, the space $\{\varphi\}$ of polymartingales of order $n$ and the space of $n$-tuples of distributions $\left\{\nu_{0}, \ldots, \nu_{n-1}\right\}$. The equivalence between the first and the third spaces was described in Theorem 4.1.

Definition 5.2. A function $\varphi$ on $T$ is a polymartingale of order $n$ or an $n$ polymartingale if $M^{n} \varphi$ is identically 0 . A second-order martingale is also called a bimartingale.

For some questions, polymartingales are more natural than polyharmonic functions. The tree version of Almansi's Theorem for polyharmonic functions (Theorem 4.1) is not as similar to the original as is the following, which is one of the main results of this section.

THEOREM 5.1. If $\varphi$ is a polymartingale of order $n$, then there exist unique martingales $\varphi_{0}, \varphi_{1}, \ldots, \varphi_{n-1}$ such that $\varphi=\sum_{k=0}^{n-1} h^{k} \varphi_{k}$, where $h$ is the length function. That is, for all $v \in T$,

$$
\varphi(v)=\sum_{k=0}^{n-1}|v|^{k} \varphi_{k}(v) .
$$

Conversely, if $\varphi_{0}, \ldots, \varphi_{n-1}$ are martingales, then $\varphi=\sum_{k=0}^{n-1} h^{k} \varphi_{k}$ is a polymartingale of order $n$.

For the proof we need the following lemma.

LEMMA 5.1. For any nonnegative integer $k$ and for any martingale $\varphi$,

$$
M h^{k} \varphi=\sum_{j=0}^{k-1}\left(\begin{array}{l}
k \\
j
\end{array}\right) h^{j} \varphi .
$$


Proof. For $v \neq e$ we have

$$
\begin{aligned}
M h^{k} \varphi(v) & =\frac{1}{q} \sum_{w^{-}=v} h^{k} \varphi(w)-h^{k} \varphi(v) \\
& =(|v|+1)^{k} \frac{1}{q} \sum_{w^{-}=v} \varphi(w)-|v|^{k} \varphi(v) \\
& =(|v|+1)^{k}(M \varphi(v)+\varphi(v))-|v|^{k} \varphi(v) \\
& =\sum_{j=0}^{k-1}\left(\begin{array}{l}
k \\
j
\end{array}\right)|v|^{j} \varphi(v)=\left(\sum_{j=0}^{k-1}(k) h^{j} \varphi\right)(v)
\end{aligned}
$$

For $v=e$, simply replace $q$ with $q+1$ and the same proof holds.

Consider the $n \times n$ matrix whose $(i, j)$-entry is $\left(\begin{array}{c}i \\ j-1\end{array}\right)$ for $j \leq i$ and zero otherwise. Note that this matrix is lower triangular with nonzero diagonal entries and hence invertible. Let $\left(b_{n, 1}, \ldots, b_{n, n}\right)$ be the bottom row of the inverse. Reindexing the formula in Lemma 5.1 it follows that $h^{n-1} \varphi=M \sum_{j=1}^{n} b_{n, j} h^{j} \varphi$ or

$$
h^{k} \varphi=M \sum_{j=1}^{k+1} b_{k+1, j} h^{j} \varphi,
$$

for any nonnegative integer $k$.

We are now ready to prove Theorem 5.1.

Proof. We use induction on $n$. The result is a tautology for $n=1$. Assume that the result holds for some $n \geq 1$, and let $\varphi$ be a polymartingale of order $n+1$. Then $M \varphi$ is a polymartingale of order $n$ so there exist martingales $\tilde{\varphi}_{0}, \ldots, \tilde{\varphi}_{n-1}$ such that $M \varphi=\sum_{k=0}^{n-1} h^{k} \tilde{\varphi}_{k}$. By (14), we have

$$
h^{k} \tilde{\varphi}_{k}=M \sum_{j=1}^{k+1} b_{k+1, j} h^{j} \tilde{\varphi}_{k}
$$

Thus $M\left(\varphi-\sum_{k=0}^{n-1} \sum_{j=1}^{k+1} b_{k+1, j} h^{j} \tilde{\varphi}_{k}\right)=0$. Let

$$
\begin{aligned}
& \varphi_{0}=\varphi-\sum_{k=0}^{n-1} \sum_{j=1}^{k+1} b_{k+1, j} h^{j} \tilde{\varphi}_{k} \text { and } \\
& \varphi_{j}=\sum_{k=j-1}^{n-1} b_{k+1, j} \tilde{\varphi}_{k} \text { for } j=1, \ldots, n .
\end{aligned}
$$


Then $\varphi_{0}, \ldots, \varphi_{n}$ are martingales and $\varphi=\sum_{j=0}^{n} h^{j} \varphi_{j}$. To prove the uniqueness, observe that if $\sum_{j=0}^{n} h^{j} \varphi_{j}$ is zero, then so is $M^{n-1} \sum_{j=0}^{n} h^{j} \varphi_{j}$, which is a nonzero multiple of $\varphi_{n}$. So $\varphi_{n}=0$. By induction, $\sum_{j=0}^{n-1} h^{j} \varphi_{j}=0$ implies $\varphi_{j}=0$ for all $j$.

We now generalize (13) to the biharmonic case. Later we shall do the same for the polyharmonic case.

THEOREM 5.2. There exist 1-1 correspondences among the space of biharmonic functions $\{f\}$, the space of bimartingales $\{\varphi\}$ and the space of pairs of distributions $\left\{\nu_{0}, \nu_{1}\right\}$ given by

$$
\begin{aligned}
\varphi & =A f, \quad f=P \nu_{0}+k P \nu_{1}, \quad \text { and } \\
\nu_{0}\left(I_{v}\right) & =\frac{1}{c_{|v|}}\left[\varphi(v)-\left(\frac{2}{q^{2}-1}+|v|\right) M \varphi(v)\right], \nu_{1}\left(I_{v}\right)=\frac{M \varphi(v)}{c_{|v|}},
\end{aligned}
$$

where

$$
A f(v)= \begin{cases}\frac{q+1}{(q-1)^{2}}(\Delta f)^{-}(v)+\frac{q}{q-1}\left(f(v)-\frac{1}{q} f^{-}(v)\right) & \text { if }|v| \geq 1 \\ \frac{2}{(q-1)^{2}} \Delta f(e)+f(e) & \text { if } v=e .\end{cases}
$$

For the proof we need the following:

LEMMA 5.2. The operator A satisfies the following properties:

(a) Iff is biharmonic, then

$$
M A f=\frac{q+1}{q-1} A \Delta f
$$

(b) For each $\omega \in \Omega, A P_{\omega}=Q_{\omega}$, where $Q_{\omega}(v)=\left\{\begin{array}{ll}c_{|v|} & \text { if } \omega \in I_{v} \\ 0 & \text { if } \omega \notin I_{v}\end{array}\right.$.

(c) For each $\omega \in \Omega, A\left(k_{\omega} P_{\omega}\right)=\left(h+\frac{2}{q^{2}-1}\right) Q_{\omega}$.

Proof. For any function $g$ on $T$ and for $|v| \geq 1$, we have

$$
M g(v)=\frac{q+1}{q} \Delta g(v)+\frac{1}{q}\left(g(v)-g^{-}(v)\right)
$$

and

$$
M g^{-}(v)=g(v)-g^{-}(v)
$$


Since $f$ is biharmonic, $\Delta f$ is harmonic and so

$$
A \Delta f(v)=\frac{q}{q-1}\left(\Delta f(v)-\frac{1}{q}(\Delta f)^{-}(v)\right) .
$$

Now for $|v| \geq 1$, using (16), (18) and (17), we obtain

$$
\begin{aligned}
\operatorname{MAf}(v)= & \frac{q+1}{(q-1)^{2}} M(\Delta f)^{-}(v)+\frac{q}{q-1}\left(M f(v)-\frac{1}{q} M f^{-}(v)\right) \\
= & \frac{q+1}{(q-1)^{2}}\left(\Delta f(v)-(\Delta f)^{-}(v)\right) \\
& +\frac{q}{q-1}\left(\frac{q+1}{q} \Delta f(v)+\frac{1}{q}\left(f(v)-f^{-}(v)\right)-\frac{1}{q}\left(f(v)-f^{-}(v)\right)\right) \\
= & \frac{q(q+1)}{(q-1)^{2}} \Delta f(v)-\frac{q+1}{(q-1)^{2}}(\Delta f)^{-}(v)=\frac{q+1}{q-1} A \Delta f(v) .
\end{aligned}
$$

Moreover

$$
\begin{aligned}
\operatorname{MAf}(e)= & \frac{1}{q+1} \sum_{|v|=1} A f(v)-A f(e) \\
= & \frac{1}{q+1} \sum_{|v|=1}\left[\frac{q+1}{(q-1)^{2}} \Delta f(e)+\frac{q}{q-1}\left(f(v)-\frac{1}{q} f(e)\right)\right] \\
& -\frac{2}{(q-1)^{2}} \Delta f(e)-f(e) \\
= & \frac{q+1}{(q-1)^{2}} \Delta f(e)+\frac{q}{q-1} \mu_{1} f(e)-\frac{1}{q-1} f(e) \\
& -\frac{2}{(q-1)^{2}} \Delta f(e)-f(e)=\frac{q+1}{q-1} \Delta f(e) \\
= & \frac{q+1}{q-1} A \Delta f(e) .
\end{aligned}
$$

Hence, for any biharmonic function $f, M A f=\frac{q+1}{q-1} A \Delta f$, proving (a).

To prove (b) observe that $A P_{\omega}(e)=1=Q_{\omega}(e)$. For $|v|=n \geq 1$,

$$
A P_{\omega}(v)=\frac{q}{q-1}\left(P_{\omega}(v)-\frac{1}{q} P_{\omega}\left(v^{-}\right)\right),
$$

since $\Delta P_{\omega}=0$. For $\omega \notin I_{v}, P_{\omega}\left(v^{-}\right)=q P_{\omega}(v)$ so $A P_{\omega}(v)=0=Q_{\omega}(v)$. For $\omega \in I_{v}$,

$$
A P_{\omega}(v)=\frac{q}{q-1}\left(q^{n}-\frac{1}{q} q^{n-1}\right)=c_{n}=Q_{\omega}(v) .
$$


Next notice that by (9),

$$
\begin{aligned}
A\left(k_{\omega} P_{\omega}\right)(e) & =\frac{2}{(q-1)^{2}} \frac{q-1}{q+1} P_{\omega}(e)+0 \cdot P_{\omega}(e) \\
& =\frac{2}{q^{2}-1}=\left(h(e)+\frac{2}{q^{2}-1}\right) Q_{\omega}(e) .
\end{aligned}
$$

For $|v|=n>0$, we have

$$
\begin{aligned}
A\left(k_{\omega} P_{\omega}\right)(v)= & \frac{q+1}{(q-1)^{2}} \frac{q-1}{q+1} P_{\omega}\left(v^{-}\right) \\
& +\frac{q}{q-1}\left(k_{\omega}(v) P_{\omega}(v)-\frac{1}{q} k_{\omega}\left(v^{-}\right) P_{\omega}\left(v^{-}\right)\right) .
\end{aligned}
$$

For $\omega \notin I_{v}$, letting $k_{\omega}(v)=k$, the right hand side of (19) becomes

$$
\frac{1}{q-1} q^{k+1}+\frac{q}{q-1}\left(k q^{k}-\frac{1}{q}(k+1) q^{k+1}\right)=0=Q_{\omega}(v)
$$

For $\omega \in I_{v}$, the right-hand side of (19) becomes

$$
\begin{aligned}
\frac{1}{q-1} q^{n-1}+\frac{q}{q-1}\left(n q^{n}-\frac{1}{q}(n-1) q^{n-1}\right) & =\left(n+\frac{2}{q^{2}-1}\right) c_{n} \\
& =\left(h(v)+\frac{2}{q^{2}-1}\right) Q_{\omega}(v),
\end{aligned}
$$

completing the proof of (c).

Proof of Theorem 5.2. Using part (a) of Lemma 5.2, we get that if $f$ is biharmonic, then

$$
M^{2} A f=\frac{q+1}{q-1} M A \Delta f=\left(\frac{q+1}{q-1}\right)^{2} A \Delta^{2} f=0
$$

proving that $A f$ is a bimartingale.

Next, assume that $\varphi$ is a bimartingale. Define $\nu_{0}, \nu_{1}$ as in (15). Since $M \varphi$ is a martingale and by Lemma 5.1 applied to $M \varphi$ with $k=1$, the function $\varphi-h M \varphi$ is also, by (13) it follows that $\nu_{0}$ and $\nu_{1}$ are distributions. Define $f=P \nu_{0}+k P \nu_{1}$, a biharmonic function. Then by Lemma 5.2 (b) and (c), recalling Notation 4.2, we have

$$
\begin{aligned}
A f & =A P \nu_{0}+A k P \nu_{1}=Q \nu_{0}+\left(h+\frac{2}{q^{2}-1}\right) M \varphi \\
& =\varphi-\left(\frac{2}{q^{2}-1}+h\right) M \varphi+\left(h+\frac{2}{q^{2}-1}\right) M \varphi=\varphi
\end{aligned}
$$


Thus $A$ is onto. To prove that $A$ is $1-1$, write $f=P \nu_{0}+k P \nu_{1}$ so that

$$
A f=Q \nu_{0}+\left(h+\frac{2}{q^{2}-1}\right) Q \nu_{1}=Q\left(\nu_{0}+\frac{2}{q^{2}-1} \nu_{1}\right)+h Q \nu_{1}
$$

But by the uniqueness result in Theorem 5.1, if $A f=0$ then $\nu_{1}=0$ and $\nu_{0}+\frac{2}{q^{2}-1} \nu_{1}=0$. Thus $\nu_{0}$ is also 0 , whence $f$ is identically 0 .

COROLlary 5.1. Given a bimartingale $\varphi$, then

$$
\begin{aligned}
A^{-1} \varphi(e)= & \varphi(e)-\frac{2}{q^{2}-1} M \varphi(e), \text { and } \\
A^{-1} \varphi(v)= & \frac{q-1}{q} \sum_{j=1}^{n} q^{-(n-j)} \varphi\left(v_{j}\right)+q^{-n} \varphi(e) \\
& -\frac{q-1}{q^{n+1}} \sum_{j=1}^{n}(n-j) q^{j} M \varphi\left(v_{j}\right)-\frac{2+n\left(q^{2}-1\right)}{q^{n}\left(q^{2}-1\right)} M \varphi(e),
\end{aligned}
$$

for $|v|=n \geq 1$, where $[e, v]=\left[v_{0}, \ldots, v_{n}\right]$.

In the special case when $f$ is a harmonic function and $\varphi$ is a martingale, formulas (16) and (20) reduce to those found in [27].

In order to prove the corollary, we shall use a general result on integration.

Lemma 5.3. Assume that for $\omega \in \Omega, K_{\omega}$ is a kernel satisfying the condition that $K_{\omega}(v)$ only depends on $k_{\omega}(v)$ for each $v \in T$. Set $K_{\omega}(v)=\alpha_{k}$ for $k_{\omega}(v)=k$. If

$$
f=K \nu=\int_{\Omega} K_{\omega} d \nu(\omega)
$$

then $f(e)=\alpha_{0} \nu(\Omega)$ and, for $|v|=n \geq 1$,

$$
f(v)=\alpha_{-n} \nu(\Omega)+\sum_{j=1}^{n}\left(\alpha_{2 j-n}-\alpha_{2 j-n-2}\right) \nu\left(I_{v_{j}}\right) .
$$

Proof. For a fixed vertex $v$ of length $n$

$$
k_{\omega}(v)= \begin{cases}n & \text { if } \omega \in I_{v} \\ 2 j-n & \text { if } \omega \in I_{v_{j}}-I_{v_{j+1}}\end{cases}
$$


Then

$$
\begin{aligned}
f(v) & =\sum_{j=0}^{n-1} \alpha_{2 j-n} \nu\left(I_{v_{j}}-I_{v_{j+1}}\right)+\alpha_{n} \nu\left(I_{v_{n}}\right) \\
& =\sum_{j=0}^{n-1} \alpha_{2 j-n} \nu\left(I_{v_{j}}\right)-\sum_{j=1}^{n} \alpha_{2 j-n-2} \nu\left(I_{v_{j}}\right)+\alpha_{n} \nu\left(I_{v_{n}}\right) \\
& =\alpha_{-n} \nu\left(I_{v_{0}}\right)+\sum_{j=1}^{n-1}\left(\alpha_{2 j-n}-\alpha_{2 j-n-2}\right) \nu\left(I_{v_{j}}\right)+\left(\alpha_{n}-\alpha_{n-2}\right) \nu\left(I_{v_{n}}\right),
\end{aligned}
$$

proving the lemma.

OBSERVATION 5.1. Let $f=\int_{\Omega} K_{\omega} d \nu(\omega)$, where

$$
K_{\omega}(v)= \begin{cases}\alpha_{|v|} & \text { for } \omega \in I_{v} \\ 0 & \text { for } \omega \notin I_{v}\end{cases}
$$

It is immediate that $f(v)=\alpha_{|v|} \nu\left(I_{v}\right)$, for all $v \in T$.

Proof of Corollary 5.1. Corresponding to the bimartingale $\varphi$, let $\nu_{0}$ and $\nu_{1}$ be the distributions defined in (15) and set $f=A^{-1} \varphi$. Then $f=P \nu_{0}+k P \nu_{1}$ so that

$$
f(e)=\nu_{0}(\Omega)=\varphi(e)-\frac{2}{q^{2}-1} M \varphi(e)
$$

and by Lemma 5.3 applied to the kernels $P_{\omega}$ and $k_{\omega} P_{\omega}$, for $v \in T$ with $|v|=n \geq 1$,

$$
\begin{aligned}
f(v)= & q^{-n} \nu_{0}(\Omega)+\sum_{j=1}^{n}\left(q^{2 j-n}-q^{2 j-n-2}\right) \nu_{0}\left(I_{v_{j}}\right) \\
& +(-n) q^{-n} \nu_{1}(\Omega)+\sum_{j=1}^{n}\left[(2 j-n) q^{2 j-n}-(2 j-n-2) q^{2 j-n-2}\right] \nu_{1}\left(I_{v_{j}}\right) \\
= & q^{-n} \nu_{0}(\Omega)+\frac{q^{2}-1}{q^{2}} \sum_{j=1}^{n} q^{2 j-n} \nu_{0}\left(I_{v_{j}}\right)-n q^{-n} \nu_{1}(\Omega) \\
& +\sum_{j=1}^{n}\left[(2 j-n) q^{2 j-n}-(2 j-n-2) q^{2 j-n-2}\right] \nu_{1}\left(I_{v_{j}}\right)=q^{-n} \varphi(e) \\
& -\frac{2 q^{-n}}{q^{2}-1} M \varphi(e)+\frac{q-1}{q} \sum_{j=1}^{n} q^{j-n}\left[\varphi\left(v_{j}\right)-\left(j+\frac{2}{q^{2}-1}\right) M \varphi\left(v_{j}\right)\right]
\end{aligned}
$$




$$
\begin{aligned}
& -n q^{-n} M \varphi(e)+\frac{q}{q+1} \sum_{j=1}^{n}\left[(2 j-n) q^{j-n}-(2 j-n-2) q^{j-n-2}\right] M \varphi\left(v_{j}\right) \\
= & \frac{q-1}{q} \sum_{j=1}^{n} q^{-(n-j)} \varphi\left(v_{j}\right)+q^{-n} \varphi(e) \\
& -\frac{q-1}{q^{n+1}} \sum_{j=1}^{n}(n-j) q^{j} M \varphi\left(v_{j}\right)-\frac{2+n\left(q^{2}-1\right)}{q^{n}\left(q^{2}-1\right)} M \varphi(e) .
\end{aligned}
$$

The correspondence $f \mapsto A f$ is not the only one between the space of biharmonic functions and the space of bimartingales. For example, given $\alpha, \beta \in \mathbb{C}$, $\alpha \neq 0$, the map $f \mapsto \alpha A f+\beta A \Delta f$ is also an isomorphism between these spaces. We shall now describe another isomorphism which turns out to be useful in allowing us to generalize Theorem 5.2 to $n$-polyharmonic functions and $n$-polymartingales.

First recall the elementary harmonic functions $P_{\omega}$ for any $\omega \in \Omega$, where $P_{\omega}(v)=q^{k_{\omega}(v)}$. Then the elementary martingales are given by $Q_{\omega}=A P_{\omega}$. Let us recall that any martingale $\varphi$ yields the distribution $\nu$, where $\nu\left(I_{v}\right)=\frac{\varphi(v)}{c_{|v|}}$. Thus we have the integral representation $\varphi=Q \nu$, since by Observation 5.1

$$
\int_{\Omega} Q_{\omega}(v) d \nu(\omega)=\int_{I_{v}} c_{|v|} d \nu(\omega)=c_{|v|} \nu\left(I_{v}\right)=\varphi(v) .
$$

We call $Q$ the martingale kernel. It follows from (13) that $\nu \mapsto Q \nu$ is a 1-1 correspondence between distributions and martingales.

Using Theorem 4.1, let $f=\sum_{m=0}^{n-1} k^{m} P \nu_{m}$ be an $n$-polyharmonic function. Define

$$
B f=\sum_{m=0}^{n-1} h^{m} Q \nu_{m} .
$$

Then $B f$ is an $n$-polymartingale and conversely, any $n$-polymartingale is of this form, by identifying $\varphi_{m}$ in Theorem 5.1 with $Q \nu_{m}$.

THEOREM 5.3. B is a 1-1 correspondence between the space of n-polyharmonic functions and the space of n-polymartingales.

Notice that if $f=P \nu$ is any harmonic function, then $B f=Q \nu=A f$, so that $B$ also generalizes the 1-1 correspondence between harmonic functions and martingales given in [27].

Let us look at the special case of $n=2$. For $f$ biharmonic, $f=P \nu_{0}+k P \nu_{1}$, and $B f=Q \nu_{0}+h Q \nu_{1}$ is a bimartingale. For any bimartingale $\varphi$, let us find explicitly the distributions $\nu_{0}$ and $\nu_{1}$ such that $\varphi=Q \nu_{0}+h Q \nu_{1}$. Since $M \varphi$ is a martingale, we may let $\nu_{1}\left(I_{v}\right)=\frac{M \varphi(v)}{c_{|v|}}$, a distribution. Thus $Q \nu_{1}=M \varphi$. The 
function $\psi=\varphi-h Q \nu_{1}$ is a martingale because

$$
M \psi=M \varphi-M h Q \nu_{1}=M \varphi-Q \nu_{1}=0,
$$

by Lemma 5.1. So, we may define the distribution $\nu_{0}$ by

$$
\nu_{0}\left(I_{v}\right)=\frac{\psi(v)}{c_{|v|}}=\frac{\varphi(v)-|v| M \varphi(v)}{c_{|v|}}
$$

That is, $Q \nu_{0}=\varphi-h M \varphi$.

This description of $B f \mapsto\left(\nu_{0}, \nu_{1}\right)$ is much simpler than that given earlier of $A f \mapsto\left(\nu_{0}, \nu_{1}\right)$. On the other hand, we do not get as simple a description of $B f$ as a function of $v$. One advantage of this method, however, is that this operator is defined for polyharmonic functions of all orders.

THEOREM 5.4. (a) Iff is a biharmonic function on $T$, then

$$
B f(v)= \begin{cases}\frac{q^{2}+1}{(q-1)^{3}} \Delta f\left(v^{-}\right)-\frac{2 q}{(q-1)^{3}} \Delta f(v)+\frac{q}{q-1}\left(f(v)-\frac{1}{q} f\left(v^{-}\right)\right) & \text {if } v \neq e, \\ f(e) & \text { if } v=e .\end{cases}
$$

(b) If $\varphi$ is a bimartingale and $v$ is a vertex of length $n$, then

$$
B^{-1} \varphi(v)= \begin{cases}\frac{\varphi(e)-n M \varphi(e)}{q^{n}}+\frac{(q-1)}{q^{n+1}} \sum_{j=1}^{n} q^{j} & {\left[\varphi\left(v_{j}\right)+\left(j-n+\frac{2}{q^{2}-1}\right) M \varphi\left(v_{j}\right)\right]} \\ & \text { if } n>0 \\ \varphi(e) & \text { if } n=0 .\end{cases}
$$

Proof. Assume $f=P \nu_{0}+k P \nu_{1}$ so that $B f=Q \nu_{0}+h Q \nu_{1}$. Using (b) and (c) of Lemma 5.2, we obtain

$$
A f=Q \nu_{0}+\left(h+\frac{2}{q^{2}-1}\right) Q \nu_{1}
$$

Thus $B f=A f-\frac{2}{q^{2}-1} Q \nu_{1}$. But $\Delta f=\frac{q-1}{q+1} P \nu_{1}$, so that $A \Delta f=\frac{q-1}{q+1} Q \nu_{1}$. Hence

$$
\frac{2}{q^{2}-1} Q \nu_{1}=\frac{2(q+1)}{\left(q^{2}-1\right)(q-1)} A \Delta f=\frac{2}{(q-1)^{2}} A \Delta f .
$$


So $B f=A f-\frac{2}{(q-1)^{2}} A \Delta f$. Consequently, for $v \neq e$

$$
\begin{aligned}
B f(v)= & \frac{q+1}{(q-1)^{2}} \Delta f\left(v^{-}\right)+\frac{q}{q-1}\left(f(v)-\frac{1}{q} f\left(v^{-}\right)\right) \\
& -\frac{2 q}{(q-1)^{3}}\left(\Delta f(v)-\frac{1}{q} \Delta f\left(v^{-}\right)\right)=\left(\frac{q+1}{(q-1)^{2}}+\frac{2}{(q-1)^{3}}\right) \Delta f\left(v^{-}\right) \\
& -\frac{2 q}{(q-1)^{3}} \Delta f(v)+\frac{q}{q-1}\left(f(v)-\frac{1}{q} f\left(v^{-}\right)\right),
\end{aligned}
$$

proving (a) for $v \neq e$. Furthermore

$$
B f(e)=A f(e)-\frac{2}{(q-1)^{2}} A \Delta f(e)=f(e),
$$

completing the proof of (a).

Next, let $\varphi=Q \nu_{0}+h Q \nu_{1}$, and set $f=B^{-1} \varphi=P \nu_{0}+k P \nu_{1}$. It is clear that $f(e)=\varphi(e)$. By Lemma 5.3, for $|v|=n>0$ we have

$$
\begin{aligned}
f(v)= & q^{-n} \nu_{0}(\Omega)+\sum_{j=1}^{n} q^{2 j-n-2}\left(q^{2}-1\right) \nu_{0}\left(I_{v_{j}}\right)-n q^{-n} \nu_{1}(\Omega) \\
& +\sum_{j=1}^{n}\left[(2 j-n) q^{2 j-n}-(2 j-n-2) q^{2 j-n-2}\right] \nu_{1}\left(I_{v_{j}}\right)=q^{-n} \varphi(e) \\
& +\left(q^{2}-1\right) \sum_{j=1}^{n} q^{2 j-n-2} \frac{\left(\varphi\left(v_{j}\right)-j M \varphi\left(v_{j}\right)\right)}{c_{j}}-n q^{-n} M \varphi(e) \\
& +\sum_{j=1}^{n}(2 j-n-2) q^{2 j-n-2}\left(q^{2}-1\right) \frac{M \varphi\left(v_{j}\right)}{c_{j}}+\sum_{j=1}^{n} 2 q^{2 j-n} \frac{M \varphi\left(v_{j}\right)}{c_{j}} \\
= & q^{-n} \varphi(e)+(q-1) \sum_{j=1}^{n} q^{j-n-1}\left(\varphi\left(v_{j}\right)-j M \varphi\left(v_{j}\right)\right)-n q^{-n} M \varphi(e) \\
& +(q-1) \sum_{j=1}^{n}\left[(2 j-n-2) q^{j-n-1}+\frac{2}{q^{2}-1} q^{j-n+1}\right] M \varphi\left(v_{j}\right) \\
= & q^{-n}(\varphi(e)-n M \varphi(e))+(q-1) \sum_{j=1}^{n} q^{j-n-1}\left[\varphi\left(v_{j}\right)\right. \\
& \left.+\left(j-n+\frac{2}{q^{2}-1}\right) M \varphi\left(v_{j}\right)\right],
\end{aligned}
$$

proving (b). 
6. Boundary behavior of polyharmonic functions. Let $T$ be a homogeneous tree of degree $q+1$. We recall that $\mu^{e}$ denotes the probability measure corresponding to the constant harmonic function 1 and the constant martingale 1. Thus $\mu^{e}\left(I_{v}\right)=\frac{1}{c_{|v|}}$, for each $v \in T$. We claim that for all vertices $v$,

$$
k P \mu^{e}(v)=|v|-\frac{2 q\left(1-q^{-|v|}\right)}{q^{2}-1} .
$$

To see this, let $f(v)=|v|-\frac{2 q\left(1-q^{-|v|}\right)}{q^{2}-1}$. Then $\Delta f$ is the constant $\frac{q-1}{q+1}$, so $f$ is biharmonic. A straightforward calculation shows that $B f=h$. On the other hand, $h=h Q \mu^{e}=B k P \mu^{e}$. Since $B$ is $1-1, f=k P \mu^{e}$.

It follows that the function $v \mapsto \frac{k P \mu^{e}}{h}(v)$ tends uniformly to 1 as $v$ approaches any boundary point. This suggests that for a distribution $\nu$ on $\Omega$ it is the boundary behavior of $\frac{k P \nu}{h}$ that should be studied. More generally, we shall discuss the boundary behavior of $\frac{k^{n} P \nu}{h^{n}}$, for any nonnegative integer $n$ with the aim of studying the boundary behavior of $\frac{f}{h^{n-1}}$ for an $n$-polyharmonic function $f$.

We first observe that we cannot expect favorable boundary behavior results for $\frac{k^{n} P \nu}{h^{n}}$ if we do not assume some restriction on $\nu$. Indeed, we now construct a distribution $\nu$ such that $\limsup _{j \rightarrow \infty} \frac{\left|k^{n} P \nu\left(\omega_{j}\right)\right|}{j^{n}}=\infty$ for every $\omega \in \Omega$ and for any $n \geq 0$, where $\left[\omega_{0}, \omega_{1}, \omega_{2}, \ldots\right)$ is the path starting at $e$ in the class $\omega$.

Example 6.1. We define a distribution $\nu$ by describing its value at each $I_{v}$ inductively on $|v|$. Let $\nu\left(I_{e}\right)=0$. Then, given the value of $\nu\left(I_{v}\right)$ with $v=w^{-}$, define $\nu\left(I_{w}\right)$ as follows:

(1) if $\nu\left(I_{v}\right)=0$, then $\nu\left(I_{w}\right)=1$ for $q-1$ of the children $w$ of $v$ and $\nu\left(I_{w}\right)=1-q$ for the remaining child $w$ of $v$;

(2) if $\nu\left(I_{v}\right)=1$, then $\nu\left(I_{w}\right)=0$ for $q-1$ of the children $w$ of $v$ and $\nu\left(I_{w}\right)=1$ for the remaining child $w$ of $v$;

(3) if $\nu\left(I_{v}\right)=1-q$, then $\nu\left(I_{w}\right)=0$ for $q-1$ of the children $w$ of $v$ and $\nu\left(I_{w}\right)=1-q$ for the remaining child $w$ of $v$.

Note that for every $\omega \in \Omega$, either $\nu\left(I_{\omega_{j}}\right)=1$ or $\nu\left(I_{\omega_{j}}\right)=1-q$ for infinitely many $j$. Also for any $v \neq e,\left|\nu\left(I_{v}\right)-\nu\left(I_{v^{-}}\right)\right| \leq q-1$. Thus, denoting [e,v] by $\left[v_{0}, v_{1}, \ldots, v_{m}\right]$, we have

$$
\begin{aligned}
\left|\sum_{j=0}^{m-1} q^{2 j-m}\left(\nu\left(I_{v_{j}}\right)-\nu\left(I_{v_{j+1}}\right)\right)\right| & \leq(q-1) q^{-m} \frac{q^{2 m}-1}{q^{2}-1} \\
& \leq \frac{q^{m}}{q+1}
\end{aligned}
$$


It follows from (11) that for any $v \in T$ for which $\nu\left(I_{v}\right)$ equals 1 or $1-q$,

$$
|P \nu(v)| \geq q^{|v|}\left|\nu\left(I_{v}\right)\right|-\frac{q^{|v|}}{q+1}
$$

Therefore $\limsup _{j \rightarrow \infty}\left|P \nu\left(\omega_{j}\right)\right|=\infty$ for every $\omega \in \Omega$. A similar analysis shows that the same conclusion holds for $\frac{k^{n} P \nu\left(\omega_{j}\right)}{j^{n}}$ for $n \in \mathbb{N}$.

In light of Example 6.1, we are going to restrict ourselves to distributions satisfying certain growth conditions.

Definition 6.1. Let $\omega \in \Omega$. We call a subset $S$ of $T$ an approach region to $\omega$ if for every $n \in \mathbb{N}$ there exists $s \in S$ such that $\omega_{n} \leq s$.

Definition 6.2. A subset $S$ of $T$ is said to be starlike with respect to $u \in T$ if for all $v \in S$, the path $[u, v]$ is contained in $S$.

Examples of approach regions which are starlike with respect to $e$ are given by the sets defined in the following.

Definition 6.3. Let $\omega \in \Omega, \tau \geq 1$ and $0 \leq a \leq \infty$. Define the approach region of $\omega$ by

$$
S_{\tau, a}(\omega)=\{v \in T:|v| \leq \tau|v \wedge \omega|+a\}
$$

Note that $S_{\tau, \infty}(\omega)=T$ for any $\tau$. If $a$ is finite, we call $S_{1, a}$ the nontangential approach region to $\omega$ of aperture $a$. If $\tau>1$, we call $S_{\tau, a}(\omega)$ the tangential approach region to $\omega$ of aperture a and tangency $\tau$. We denote by $S_{\tau}(\omega)$ the approach region $S_{\tau, 0}(\omega)$.

Remark 6.1. We can define a distance $\rho$ on $T$ by

$$
\rho(u, v)=\left\{\begin{array}{ll}
q^{-|u \wedge v|}, & \text { for } u \neq v \\
0 & \text { for } u=v
\end{array} .\right.
$$

The completion of $T$ with respect to $\rho$ is exactly $T \cup \Omega$. Observe that the $\rho$-balls in $\Omega$ are exactly the sets $I_{v}$, for $v \in T$, and $I_{v}$ has $\rho$-radius $q^{-|v|}$. For $\tau>1$, $S_{\tau, a}(\omega)$ is tangential in the sense that there exists a constant $c\left(c=q^{a}\right)$ such that for any $v \in S_{\tau, a}(\omega)$

$$
\rho(v, \omega)^{\tau} \leq c \rho(v, \Omega)
$$

This is the precise analogue of the classical tangential approach region to a boundary point in the upper half plane, where $\rho$ represents Euclidean distance. 
Definition 6.4. Let $S$ be an approach region of $\omega \in \Omega$. Let $[e, \omega)=\left[\omega_{0}, \omega_{1}, \ldots\right)$ as above. A function $f: T \rightarrow \mathbb{C}$ is said to have $S$-limit $L$ at $\omega$ if for all $\epsilon>0$ there exists $m \in \mathbb{N}$ such that $|f(v)-L|<\epsilon$ for all $v \in S$ with $v \geq \omega_{m}$. We denote this limit by $\lim _{S, \omega} f$. If $\lim _{T, \omega} f$ exists we say that $f$ has an unrestricted limit at $\omega$. If for some $\tau \geq 1, \lim _{S, \omega} f$ exists for $S=S_{\tau, a}(\omega)$ for all $a<\infty$ (necessarily independent of $a$ ), then we call this limit the $\tau$-limit of $f$ at $\omega$ and we denote it by $\tau$ - $\lim _{\omega} f$. 1-limits are also referred to as nontangential limits.

The following proposition will allow us to deduce boundary behavior results for $n$-polyharmonic functions from boundary behavior results for martingales.

PROPOSITION 6.1. Let $\mu$ be a distribution on $\Omega$ and let $S$ be an approach region to $\omega \in \Omega$ which is starlike with respect to e. Suppose that $Q \mu$ has $S$-limit $L$ at $\omega$. Then $\frac{k^{n} P \mu}{h^{n}}$ also has $S$-limit $L$ at $\omega$ for each nonnegative integer $n$.

Proof. Fix $n \geq 0$ and let $0<\epsilon<1 / 3$. Since $Q \mu$ has $S$-limit $L$ at $\omega$, there exists $N \in \mathbb{N}$ such that $\left|c_{|v|} \mu\left(I_{v}\right)-L\right|<\epsilon$ for all $v \in S$, with $v \geq \omega_{N}$. Let

$$
M=\max \left\{|L|+\epsilon, c_{j}\left|\mu\left(I_{\omega_{j}}\right)\right|, j=1, \ldots, N\right\} .
$$

For $m>\frac{N}{1-\epsilon}$, let $J_{m}=\lfloor m(1-\epsilon)\rfloor$ so that $J_{m} \geq N$. Fix $v \geq \omega_{N}$. Setting $[e, v]=$ $\left[v_{o}, \ldots, v_{m}\right]$ as usual, let $I_{j}$ denote $I_{v_{j}}$, which is $I_{\omega_{j}}$ for $j \leq N$. By Lemma 5.3, we have

$$
\begin{aligned}
\frac{k^{n} P \mu}{h^{n}}(v)= & (-1)^{n} q^{-m} \mu(\Omega)+\sum_{j=1}^{m} \frac{q^{2 j-m}}{m^{n}}\left[(2 j-m)^{n}-\frac{(2 j-m-2)^{n}}{q^{2}}\right] \mu\left(I_{j}\right) \\
= & (-1)^{n} q^{-m} \mu(\Omega)+\sum_{j=1}^{m} q^{2 j-m} \frac{q^{2}-1}{q^{2}}\left(\frac{2 j-m}{m}\right)^{n} \mu\left(I_{j}\right) \\
& -\sum_{k=0}^{n-1}\left(\begin{array}{l}
n \\
k
\end{array}\right) \sum_{j=1}^{m} q^{2 j-m} \frac{(2 j-m)^{k}(-2)^{n-k}}{m^{n} q^{2}} \mu\left(I_{j}\right) \\
= & (-1)^{n} q^{-m} \mu(\Omega)+\sum_{j=1}^{J_{m}-1} \frac{q-1}{q} q^{j-m}\left(\frac{2 j-m}{m}\right)^{n} c_{j} \mu\left(I_{j}\right) \\
& +\sum_{j=J_{m}}^{m} \frac{q-1}{q} q^{j-m}\left(\frac{2 j-m}{m}\right)^{n} c_{j} \mu\left(I_{j}\right) \\
& -\sum_{k=0}^{n-1}\left(\begin{array}{l}
n \\
k
\end{array}\right)(-2)^{n-k} \sum_{j=1}^{m} q^{j-m} \frac{(2 j-m)^{k}}{m^{n} q(q+1)} c_{j} \mu\left(I_{j}\right) \\
= & I+\frac{q-1}{q} I I+I I I-\sum_{k=0}^{n-1}\left(\begin{array}{l}
n \\
k
\end{array}\right) \frac{(-2)^{n-k}}{q(q+1)} I V_{k} .
\end{aligned}
$$


We shall show that $I, I I$, and each $I V_{k}$ approach 0 and $I I I$ approaches $L$ as $m \rightarrow \infty$. It is clear that $I$ goes to 0 . Using the three facts that $\left|c_{j} \mu\left(I_{j}\right)\right| \leq M$ for all $j$, $\sum_{j=1}^{m} q^{j-m}<\frac{q}{q-1}$ and $\left|\frac{(2 j-m)^{k}}{m^{n}}\right| \leq m^{k-n}$ for $j=1, \ldots, m$, it follows that for each $k=0, \ldots, n-1$,

$$
\left|I V_{k}\right|<\frac{q}{q-1} m^{k-n} M<\frac{2 M}{m}
$$

and thus $I V_{k} \rightarrow 0$ as $m \rightarrow \infty$. Moreover

$$
|I I| \leq \sum_{j=1}^{J_{m}-1} q^{j-m} M<\frac{q^{J_{m}-m}}{q-1} M .
$$

Observing that $J_{m}-m \leq-m \epsilon$, we see that $I I \rightarrow 0$ as $m \rightarrow \infty$. Next we look at III $-L$.

$$
\begin{aligned}
|I I I-L| \leq & \sum_{j=J_{m}}^{m} \frac{q-1}{q} q^{j-m}\left|\frac{2 j-m}{m}\right|^{n}\left|c_{j} \mu\left(I_{j}\right)-L\right| \\
& +|L| \sum_{j=J_{m}}^{m} \frac{q-1}{q} q^{j-m}\left|\left(\frac{2 j-m}{m}\right)^{n}-1\right|+|L|\left|\sum_{j=J_{m}}^{m} \frac{q-1}{q} q^{j-m}-1\right| \\
\leq & \sum_{j=0}^{m} \frac{q-1}{q} q^{j-m} \epsilon+|L| \sum_{j=J_{m}}^{m} \frac{q-1}{q} q^{j-m}\left[1-\left(\frac{2 j-m}{m}\right)^{n}\right]+\frac{|L|}{q^{m \epsilon}} .
\end{aligned}
$$

Notice that since $J_{m} \leq j \leq m, 2 j-m \geq 2 J_{m}-m \geq m-2 m \epsilon-2$, so

$$
\frac{2 j-m}{m} \geq 1-2 \epsilon-\frac{2}{m} \geq 1-3 \epsilon
$$

for $m$ large. Thus

$$
1-\left(\frac{2 j-m}{m}\right)^{n}<1-(1-3 \epsilon)^{n} \leq 3 n \epsilon
$$

Hence $|I I I-L| \leq \epsilon+|L| 3 n \epsilon+|L| q^{-m \epsilon}$. Thus

$$
\lim _{m \rightarrow \infty}|I I I-L| \leq \epsilon(1+3 n|L|)
$$

But this is true for all $\epsilon \in(0,1 / 3)$, and thus $I I I \rightarrow L$ as $m \rightarrow \infty$.

Let $\mu$ be a complex Borel measure on $\Omega$ and let $\varphi=Q \mu$ be the corresponding martingale. It is known that $\varphi$ has a nontangential limit at $\mu^{e}$-a.e. boundary point. Suppose in addition that $\mu$ is $\mu^{e}$-absolutely continuous with density function $g$. 
It is also known that if $g$ is continuous at $\omega \in \Omega$, then $\varphi$ has an unrestricted limit at $\omega$. For a discussion of these results see [27]. Combining these facts with Proposition 6.1 we deduce:

THEOREM 6.1. Let $f$ be n-polyharmonic on $T$. Let $\nu_{0}, \ldots, \nu_{n-1}$ be the corresponding distributions of Theorem 4.1.

(a) If $\nu_{0}, \ldots, \nu_{n-1}$ are complex Borel measures, then $\frac{f}{h^{n-1}}$ has a nontangential limit at $\mu^{e}$-a.e. boundary point of $\Omega$.

(b) Suppose that $\nu_{0}, \ldots, \nu_{n-1}$ are $\mu^{e}$-absolutely continuous measures whose density functions are continuous at $\omega \in \Omega$, then $\frac{f}{h^{n-1}}$ has an unrestricted limit at $\omega$.

We now consider boundary behavior results of $n$-polyharmonic functions which include both nontangential and tangential approach regions as well as exceptional sets which are thinner than those of $\mu^{e}$-measure zero. In order to describe these exceptional sets, we recall the definition of the Hausdorff measures.

Definition 6.5. Let $E$ be a subset of $\Omega$. Let $0<\beta \leq 1$. We define

$$
H_{\beta}(E)=\sup _{\delta>0} \inf _{A \subset T}\left\{\sum_{v \in A} \rho(v, \Omega)^{\beta}: E \subset \bigcup_{v \in A} I_{v}, \rho(A, \Omega)<\delta\right\} .
$$

We call $H_{\beta}(E)$ the $\beta$-dimensional Hausdorff measure of $E$. We say that a property holds $\beta$-a.e. if it holds for all $\omega$ except for those in a subset of $\Omega$ having $H_{\beta}$ measure 0 .

The following two lemmas, the second of which is of Frostman type, were proved in [19].

LemMa 6.1. A Borel subset of $\Omega$ has $H_{\beta}$ measure 0 if and only if every compact subset of it has $\mathrm{H}_{\beta}$ measure 0 .

LEMMA 6.2. Let $K$ be a compact subset of $\Omega$ having positive $H_{\beta}$ measure. Then there exists a measure $\nu$ on $\Omega$ supported on $K$ such that $0<\nu(K)<\infty$ and for every $v \in T, \nu\left(I_{v}\right) \leq q^{-\beta|v|}$.

The following space of functions is fundamental in our study of boundary behavior.

Definition 6.6. For $\alpha \geq 0$, let $\mathcal{A}_{\alpha}$ be the space consisting of all complexvalued functions $f$ on $T$ satisfying the condition

$$
\sum_{v \in T}|f(v)| q^{-(1-\alpha)|v|}<\infty
$$

Throughout the remainder of this section we fix once and for all constants $\alpha$ and $\tau$ where $0 \leq \alpha<1$ and $1 \leq \tau \leq 1 /(1-\alpha)$. 
The letter $c$ will be used to represent a constant value which, though possibly different with each occurrence, does not depend on the parameters of interest.

The following lemma will be used to derive a general boundary behavior result.

Lemma 6.3. Let $f \in \mathcal{A}_{\alpha}$. Fix $a \in[0, \infty)$ and define $F: \Omega \rightarrow[0, \infty]$, by

$$
F(\omega)=\sum_{v \in S_{\tau, a}(\omega)}|f(v)| .
$$

Then $F(\omega)$ is finite $\tau(1-\alpha)$-a.e.

Proof. Without loss of generality, we may assume that $f$ is a nonnegative (real-valued) function. Define the sequence $\left\{F_{n}\right\}_{n=0}^{\infty}$ of functions on $\Omega$ by

$$
F_{n}(\omega)=\sum_{\substack{v \geq \omega_{n} \\|v| \leq n \tau+a}} f(v) .
$$

Note that $F(\omega) \leq \sum_{n=0}^{\infty} F_{n}(\omega)$ for all $\omega \in \Omega$. Moreover, each $F_{n}$ is locally constant hence continuous. Thus $\sum_{n=0}^{\infty} F_{n}(\omega)$ is Borel measurable. We shall prove that $\sum_{n=0}^{\infty} F_{n}(\omega)$ is finite $\tau(1-\alpha)$-a.e. Arguing by contradiction, assume that the set

$$
\left\{\omega \in \Omega: \sum_{n=0}^{\infty} F_{n}(\omega)=\infty\right\}
$$

has positive $H_{\beta}$ measure. Then by Lemma 6.1, there is a compact subset $K$ of positive $H_{\beta}$ measure. So by Lemma 6.2, we can find a Borel measure $\nu$ that is supported on $K$ and satisfies the condition

$$
\nu\left(I_{v}\right) \leq q^{-(1-\alpha) \tau|v|} \text { for all } v \in T .
$$

We then get a contradiction if we show that

$$
\int_{\Omega} \sum_{n=0}^{\infty} F_{n}(\omega) d \nu(\omega)<\infty .
$$

Since each $F_{n}$ is nonnegative, we can interchange the order of integration and summation and obtain

$$
\begin{aligned}
\int_{\Omega} \sum_{n=0}^{\infty} F_{n}(\omega) d \nu(\omega) & =\sum_{n=0}^{\infty} \int_{\Omega} F_{n}(\omega) d \nu(\omega) \\
& =\sum_{n=0}^{\infty} \sum_{|\omega|=n} \int_{I_{w}} F_{n}(\omega) d \nu(\omega)
\end{aligned}
$$




$$
\begin{aligned}
& \leq \sum_{n=0}^{\infty} \sum_{|w|=n} \sum_{\substack{v \geq w \\
|v| \leq n \tau+a}} f(v) q^{-\tau(1-\alpha) n} \\
& =\sum_{w \in T} \sum_{\substack{v \geq w \\
|v| \leq \tau|w|+a}} f(v) q^{-(1-\alpha) \tau|w|} \\
& =\sum_{v \in T} f(v) \sum_{\substack{w \leq v \\
|w| \geq(|v|-a) / \tau}} q^{-(1-\alpha) \tau|w|} \\
& \leq \sum_{v \in T} f(v) \sum_{n \geq(|v|-a) / \tau} q^{-(1-\alpha) \tau n} \\
& \leq c \sum_{v \in T} f(v) q^{-(1-\alpha) \tau|v| / \tau} \\
& =c \sum_{v \in T} f(v) q^{-(1-\alpha)|v|},
\end{aligned}
$$

which is finite. This completes the proof.

Definition 6.7. Let $\varphi$ be a complex-valued function on $T$. Define

$$
D \varphi(v)= \begin{cases}\varphi(v)-\varphi\left(v^{-}\right) & \text {for } v \neq e \\ 0 & \text { for } v=e .\end{cases}
$$

We call $D \varphi(v)$ the inner normal derivative of $\varphi$ at $v$ if $v \neq e$.

Corollary 6.1. Let $\varphi$ be any function on $T$ such that $D \varphi \in \mathcal{A}_{\alpha}$. Then $\varphi$ has $\tau$-limits $\tau(1-\alpha)$-a.e.

Proof. Fix $a \geq 0$. Then by Lemma 6.3, we have

$$
\sum_{v \in S_{\tau, a}(\omega)}|D \varphi(v)|<\infty
$$

$\tau(1-\alpha)$-a.e. Let $\omega$ be any point for which this sum is finite. Let $\epsilon>0$ and choose a positive integer $N$ so that

$$
\sum_{v \in S_{\tau, a}(\omega),|v| \geq N}|D \varphi(v)|<\epsilon
$$

Let $u_{1}, u_{2}$ be any two vertices in $S_{\tau, a}(\omega)$ of length greater than or equal to $\tau N+a$. This implies that $\left[u_{1}, u_{2}\right] \subset\left\{v \in S_{\tau, a}(\omega):|v| \geq N\right\}$. Let $\left[u_{1}, u_{2}\right]=\left[v_{0}, v_{1}, \ldots, v_{n}\right]$. Then

$$
\begin{aligned}
\left|\varphi\left(u_{1}\right)-\varphi\left(u_{2}\right)\right| & \leq \sum_{i=1}^{n}\left|D \varphi\left(v_{i}\right)\right| \\
& \leq \sum_{\substack{v \in S_{\tau, a}(\omega) \\
|v| \geq N}}|D \varphi(v)| \\
& <\epsilon .
\end{aligned}
$$


Thus if $\left\{u_{j}\right\}$ is any sequence approaching $\omega$ in $S_{\tau, a}(\omega)$, then $\left\{\varphi\left(u_{j}\right)\right\}$ is a Cauchy sequence. Thus $\lim _{j \rightarrow \infty} \varphi\left(u_{j}\right)$ exists and is finite.

PROPOSITION 6.2. Let $\nu$ be a distribution on $\Omega$. Let $f=P \nu$ be the corresponding harmonic function on $T$ and let $\varphi=Q \nu$ be the corresponding martingale. Then the following conditions are equivalent:

(a) $\sum_{v \neq e}\left|\nu\left(I_{v}\right)-\frac{1}{q} \nu\left(I_{v^{-}}\right)\right| q^{\alpha|v|}<\infty$;

(b) $D f \in \mathcal{A}_{\alpha}$;

(c) $D \varphi \in \mathcal{A}_{\alpha}$.

Proof. First observe that (a) is equivalent to (c) since $\varphi(v)=c_{|v|} \nu\left(I_{v}\right)$ for all $v \in T$, so that

$$
\begin{aligned}
\sum_{v \neq e}|D \varphi(v)| q^{-(1-\alpha)|v|} & =\sum_{v \neq e}\left|c_{|v|} \nu\left(I_{v}\right)-c_{|v|-1} \nu\left(I_{v^{-}}\right)\right| q^{-(1-\alpha)|v|} \\
& =\frac{q+1}{q} \sum_{v \neq e}\left|\nu\left(I_{v}\right)-\frac{1}{q} \nu\left(I_{v^{-}}\right)\right| q^{\alpha|v|} .
\end{aligned}
$$

Using (13) we deduce

$$
\nu\left(I_{v}\right)-\frac{1}{q} \nu\left(I_{v^{-}}\right)=\frac{q^{2}}{q^{2}-1} \frac{D f(v)-\frac{1}{q} D f\left(v^{-}\right)}{q^{|v|}}, v^{-} \neq e,
$$

showing that (b) implies (a).

To complete the proof we show that (c) implies (b). Let $v \in T$ be such that $|v|=n \geq 1$. Again using (13) we derive the relation

$$
D f(v)=\frac{q-1}{q} \sum_{j=1}^{n} q^{-(n-j)} D \varphi\left(v_{j}\right)
$$

Thus

$$
\begin{aligned}
\sum_{v \in T}|D f(v)| q^{-(1-\alpha)|v|} & =\sum_{n=1}^{\infty} \sum_{|v|=n}|D f(v)| q^{-(1-\alpha) n} \\
& \leq \sum_{n=1}^{\infty} \sum_{|v|=n} \sum_{j=1}^{n} q^{-(n-j)}\left|D \varphi\left(v_{j}\right)\right| q^{-(1-\alpha) n} \\
& =\sum_{j=1}^{\infty} \sum_{|v|=j} \sum_{n=j}^{\infty}|D \varphi(v)| q^{-(1-\alpha) n}
\end{aligned}
$$




$$
\begin{aligned}
& =\sum_{j=1}^{\infty} \sum_{|v|=j}|D \varphi(v)| \frac{q^{-(1-\alpha) j}}{1-q^{-(1-\alpha)}} \\
& =\sum_{v \neq e}|D \varphi(v)| \frac{q^{-(1-\alpha)|v|}}{1-q^{-(1-\alpha)}},
\end{aligned}
$$

completing the proof.

Definition 6.8. Let $\mathcal{B}_{\alpha}$ be the space of distributions $\nu$ on $\Omega$ satisfying the condition

$$
\sum_{v \neq e}\left|\nu\left(I_{v}\right)-\frac{1}{q} \nu\left(I_{v^{-}}\right)\right| q^{\alpha|v|}<\infty
$$

Let $\mathcal{H}_{\alpha}^{n}$ be the space of $n$-polyharmonic functions such that the corresponding distributions of Theorem 4.1 are in $\mathcal{B}_{\alpha}$.

Remark 6.2. The space $\mathcal{B}_{\alpha}$ is precisely the Besov-Lipschitz space $B_{1,1}^{\alpha}$ defined in [13]. In light of Proposition 6.2, this Besov-Lipschitz space can be identified with the space of all harmonic functions in $\mathcal{H}_{\alpha}^{1}$, or equivalently, the space of martingales whose inner normal derivative is in $\mathcal{A}_{\alpha}$.

We now deduce our main boundary behavior result for $n$-polyharmonic functions.

THEOREM 6.2. Let $0 \leq \alpha<1,1 \leq \tau \leq 1 /(1-\alpha)$. Letf be $n$-polyharmonic on $T$ and let $\nu_{0}, \ldots, \nu_{n-1}$ be the corresponding distributions of Theorem 4.1. Suppose $f \in \mathcal{H}_{\alpha}^{n}$. Then $\frac{f}{h^{n-1}}$ has $\tau$-limits $\tau(1-\alpha)$-a.e. and these limits are equal to the $\tau$-limits of $P \nu_{n-1}$.

Proof. Using Proposition 6.2, we may apply Corollary 6.1 to each martingale $\varphi_{j}=Q \nu_{j}$. The conclusion now follows easily from Proposition 6.1.

Remark 6.3. Let $\nu$ be a distribution in $\mathcal{B}_{\alpha}$ and let $f$ be the corresponding harmonic function $P \nu$. Despite the fact that $\frac{k P \nu}{h}$ has $\tau$-limits $\tau(1-\alpha)$-a.e., it is not in general true that the inner normal derivative of $\frac{k P \nu}{h}$ is in $\mathcal{A}_{\alpha}$. Indeed, from (21) for $|v| \geq 2$ we obtain

$$
\left|D\left(\frac{k P \mu^{e}(v)}{|v|}\right)\right|=\frac{2 q}{q^{2}-1}\left|\frac{1-q^{-n}-n q^{-n}(q-1)}{n(n-1)}\right| .
$$

Thus

$$
\sum_{v, v^{-} \neq e}\left|D\left(\frac{k P \mu^{e}(v)}{|v|}\right)\right| q^{-(1-\alpha)|v|}=\sum_{n=2}^{\infty} \frac{2 q^{\alpha n}}{n(n-1)}\left|\frac{1-q^{-n}}{q-1}-n q^{-n}\right|
$$


which is divergent. Thus, there appears to be no simpler description of the space $\mathcal{H}_{\alpha}^{n}$.

We now show that the exceptional set and the size of the approach region in Theorem 6.2 are the best possible.

THEOREM 6.3. (a) Let $0 \leq \alpha<1$ and $1 \leq \tau \leq 1 /(1-\alpha)$. Let $E$ be a subset of $\Omega$ such that $H_{\tau(1-\alpha)}(E)=0$. Then there is a harmonic function $f \in \mathcal{H}_{\alpha}^{1}$ such that for all $\omega \in E, \lim \sup f=\infty$.

$$
S_{\tau}(\omega)
$$

(b) Let $0 \leq \alpha<1$ and $\tau>1 /(1-\alpha)$. Then there is a harmonic function $f \in \mathcal{H}_{\alpha}^{1}$ such that $\limsup _{S_{\tau}(\omega)} f=\infty$ for all $\omega \in \Omega$.

Proof. (a) For each positive integer $i$ there exists a sequence of vertices $\left\{v_{i j}\right\}_{j}$ such that $\left|v_{i j}\right|>i, E \subset \bigcup_{j} I_{v_{i j}}$ and $\sum_{j} q^{-\tau(1-\alpha)\left|v_{i j}\right|}<2^{-i}$. For each vertex $v_{i j}$ we associate a vertex $u_{i j} \geq v_{i j}$ such that $\left|u_{i j}\right|=\left\lfloor\tau\left|v_{i j}\right|\right\rfloor$. Let $M_{i j}=i q^{-\tau\left|v_{i j}\right|}$. Let $\nu_{i j}$ be the positive distributions defined as follows: for each $v \in T$

$$
\nu_{i j}\left(I_{v}\right)= \begin{cases}0 & \text { if } I_{v} \cap I_{u_{i j}}=\emptyset \\ q^{\left|u_{i j}\right|-|v|} M_{i j} & \text { if } v \geq u_{i j} \\ M_{i j} & \text { if } v<u_{i j} .\end{cases}
$$

From the definitions we see that $\sum_{i, j} \nu_{i, j}\left(I_{v}\right)$ converges for all vertices $v$. So, letting $\nu=\sum_{i, j} \nu_{i j}$, it follows that $\nu$ is a positive distribution. Let $f=P \nu$, a harmonic function. Note that

$$
\sum_{|v| \geq 1}\left|\nu_{i j}\left(I_{v}\right)-\frac{1}{q} \nu_{i j}\left(I_{v^{-}}\right)\right| q^{\alpha|v|} \leq c M_{i j} \sum_{n=0}^{\left|u_{i j}\right|} q^{\alpha n} \leq c M_{i j} q^{\alpha\left|u_{i j}\right|}
$$

Thus

$$
\begin{aligned}
\sum_{|v| \geq 1}\left|\nu\left(I_{v}\right)-\frac{1}{q} \nu\left(I_{v^{-}}\right)\right| q^{\alpha|v|} & \leq c \sum_{i, j} M_{i j} q^{\alpha\left|u_{i j}\right|} \leq c \sum_{i, j} M_{i j} q^{\alpha \tau\left|v_{i j}\right|} \\
& =c \sum_{i, j} i q^{-(1-\alpha) \tau\left|v_{i j}\right|}<c \sum_{i} \frac{i}{2^{i}}<\infty
\end{aligned}
$$

so by Proposition 6.2, $\sum_{v \in T}|D f(v)| q^{-(1-\alpha)|v|}$ is finite. Also

$$
f\left(u_{i j}\right)=P \nu\left(u_{i j}\right) \geq q^{\left|u_{i j}\right|} \nu_{i j}\left(I_{u_{i j}}\right) \geq \frac{1}{q} q^{\tau\left|v_{i j}\right|} M_{i j}=\frac{1}{q} i .
$$


Since for any $\omega \in E$ there are integers $i$ and $j$ with $i$ arbitrarily large such that $u_{i j} \in S_{\tau(1-\alpha)}(\omega)$, we are done.

(b) Choose $\epsilon$ such that $0<\epsilon<\tau(1-\alpha)-1$. Define

$$
M_{i j}=q^{-(\alpha \tau+1+\epsilon) i} \quad \text { for each } j=1, \ldots,(q+1) q^{i-1} .
$$

Let $\left\{v_{i j}\right\}_{j}$ be the collection of all the vertices of length $i$. Let $u_{i j}$ be any vertex of length $\lfloor\tau i\rfloor$ such that $u_{i j} \geq v_{i j}$. Define $\nu_{i j}, \nu$ and $f$ as in the proof of part (a). Then

$$
\sum_{|v| \geq 1}\left|\nu\left(I_{v}\right)-\frac{1}{q} \nu\left(I_{v^{-}}\right)\right| q^{\alpha|v|} \leq c \sum_{i=0}^{\infty} q^{-(\alpha \tau+1+\epsilon) i} q^{\alpha i \tau} q^{i}=c \sum_{i=0}^{\infty} q^{-\epsilon i}
$$

which is finite, and

$$
\begin{aligned}
P \nu\left(u_{i j}\right) & \geq q^{-(\alpha \tau+1+\epsilon) i} q^{\left|u_{i j}\right|} \geq c q^{-(\alpha \tau+1+\epsilon) i} q^{\tau i} \\
& =c q^{i(\tau-\alpha \tau-1-\epsilon)}=c q^{i(\tau(1-\alpha)-1-\epsilon)} \rightarrow \infty \text { as } i \rightarrow \infty .
\end{aligned}
$$

Department of Mathematics, University of Maryland, College Park, MD 20742 4015

E-mail: jmc@math.umd.edu

DePARTMENT of MATHEMATICS, GeORge MASON University, FAIRFAX, VA 22030

E-mail: fcolonna@gmu.edu

Department of Mathematics, Mcgill University, Montreal, Quebec, Canada H3A 2K6

E-mail: gowri@math.mcgill.ca

Department of Mathematics, GeORge Mason University, FairfaX, VA 22030

E-mail: dsingman@gmu.edu

REFERENCES

[1] E. Almansi, Sull'integrazione dell'equazione differenziale $\Delta^{2} \Delta^{2}=0$, Atti Accad. Sci. Torino Cl. Sci. Fis. Mat. Natur. 31 (1896), 881-888.

[2] Sull'integrazione dell'equazione differenziale $\Delta^{2 n}=0$, Ann. Mat. Pura Appl. (III) II (1899), $1-59$.

[3] L. E. Andersson, T. Elfving, and G. H. Golub, Solution of biharmonic equations with application to radar imaging, J. Comput. Appl. Math. 94 (1998), 153-180. 
[4] N. Aronszajn, T. M. Creese, and L. J. Lipkin, Polyharmonic Functions, Oxford Math. Monogr., Oxford University Press, New York, 1983.

[5] C. A. Berenstein, E. Casadio Tarabusi, J. M. Cohen, and M. A. Picardello, Integral geometry on trees, Amer. J. Math. 113 (1991), 441-470.

[6] W. L. Briggs and V. Henson, The DFT, Soc. Ind. Appl. Math., Philadelphia, 1995.

[7] P. Cartier, Fonctions harmoniques sur un arbre, Sympos. Math., vol. 9, Cambridge University Press, Cambridge, 1972, pp. 203-270.

[8] E. Casadio Tarabusi, J. M. Cohen, and F. Colonna, Characterization of the range of the Radon transform on homogeneous trees, Electron. Res. Announc. Amer. Math. Soc. 5 (1999), 11-17.

[9] The range of the horocyclic Radon transform on trees, Ann. Inst. Fourier (Grenoble) $\mathbf{5 0}$ (2000), 211-234.

[10] E. Casadio Tarabusi, J. M. Cohen, A. Korányi, and A. M. Picardello, Converse mean value theorems on trees, J. Lie Theory 8 (1998), 229-254.

[11] E. Casadio Tarabusi, J. M. Cohen, and A. M. Picardello, Range of the X-ray transform on trees, Adv. Math. 109 (1994), 153-167.

[12] J. M. Cohen and F. Colonna, The functional analysis of the X-ray transform on trees, Adv. in Appl. Math. 14 (1993), 123-138.

[13] _ Extreme points of the Bloch space of a homogeneous tree, Israel J. Math. 94 (1996), 247271.

[14] J. M. Cohen and A. M. Picardello, The 2-circle and 2-disk problems on trees, Israel J. Math. 64 (1988), 73-86.

[15] F. Di Biase, Fatou type Theorems. Maximal Functions and Approach Regions, Progr. Math., vol. 147, Birkhäuser, Boston, 1998.

[16] K. Dalrymple, R. S. Strichartz, and J. P. Vinson, Fractal differential equations on the Sierpinski gasket, J. Fourier Anal. Appl. 5 (1999), 203-284.

[17] A. Figà-Talamanca and M. A. Picardello, Harmonic Analysis on Free Groups, Lecture Notes in Pure and Appl. Math., vol. 87, Marcel Dekker, New York, Basel, 1983.

[18] J. Globevnik and W. Rudin, A characterization of harmonic functions, Indag. Math. 50 (1988), 419-426.

[19] K. GowriSankaran and D. Singman, Tangential limits of potentials on homogeneous trees, Potential Anal. (to appear).

[20] J. Kigami, Harmonic calculus on the Sierpinski gasket, Japan J. Appl. Math. 6 (1989), 259-290.

[21] A. Korányi and M. A. Picardello, Boundary behaviour of eigenfunctions of the Laplace operator on trees, Ann. Sci. Norm. Sup. Pisa 13 (1986), 389-399.

[22] S. A. Lurie and V. V. Vasiliev, The Biharmonic Problem in the Theory of Elasticity, Gordon and Breach Pub., Amsterdam, 1995.

[23] E. Mathieu, Sur l'équation aux différences partielles du quatrième ordre $\Delta \Delta u=0$ et sur l'équilibre d'élasticité d'un corps solide, Journal de Math., $2^{e}$ série, t. XIV (1869), 378-421.

[24] Y. Mizuta, Potential Theory in Euclidean Spaces, Gakuto Internat. Ser. Math. Sci. Appl., Gakk-otasho, Tokyo, 1996.

[25] M. Nicolesco, Les Fonctions Polyharmoniques, Actualités Sci. Indust., vol. 331, Hermann, Paris, 1936.

[26] C. Riquier, Sur quelques problèmes relatifs a l'équation aux derivées partielles $\Delta(u)=0$, Journal de Math. (1926), 297.

[27] M. H. Taibleson, Hardy spaces of harmonic functions on homogeneous isotropic trees, Math. Nach. 133 (1987), 273-288. 